\title{
An explicit meshless point collocation method for electrically driven magnetohydrodynamics (MHD) flow
}

\author{
G. C. Bourantas ${ }^{1}$, V. C. Loukopoulos ${ }^{2}$, G. R. Joldes ${ }^{1,3}$, A. Wittek ${ }^{1}$, K. Miller ${ }^{1,4}$ \\ ${ }^{I}$ Intelligent Systems for Medicine Laboratory, School of Mechanical and Chemical Engineering, The University \\ of Western Australia, 35 Stirling Highway, Crawley-Perth, WA 6009, Australia \\ ${ }^{2}$ Department of Physics, University of Patras, Patras, GR 26500, Rion, Greece. \\ ${ }^{3}$ School of Engineering and Information Technology, Murdoch University, 90 South St, Murdoch, Australia \\ ${ }^{4}$ School of Engineering, Cardiff University, The Parade, CF24 3AA Cardiff, United Kingdom
}

\begin{abstract}
In this paper, we develop a meshless collocation scheme for the numerical solution of magnetohydrodynamics (MHD) flow equations. We consider the transient laminar flow of an incompressible, viscous and electrically conducting fluid in a rectangular duct. The flow is driven by the current produced by electrodes placed on the walls of the duct. The method combines a meshless collocation scheme with the newly developed Discretization Corrected Particle Strength Exchange (DC PSE) interpolation method. To highlight the applicability of the method, we discretize the spatial domain by using uniformly (Cartesian) and irregularly distributed nodes. The proposed solution method can handle high Hartmann $(\mathrm{Ha})$ numbers and captures the boundary layers formed in such cases, without the presence of unwanted oscillations, by employing a local mesh refinement procedure close to the boundaries. The use of local refinement reduces the computational cost. We apply an explicit time integration scheme and we compute the critical time step that ensures stability through the Greshgorin theorem. Finally, we present numerical results obtained by using different orientation of the applied magnetic field.
\end{abstract}

Keywords: Meshless Point Collocation, Discretization Corrected Particle Strength Exchange (DC PSE), unsteady MHD, Hartmann number, Explicit.

\section{Introduction}

In this paper, we apply a meshless point collocation (MPC) scheme to numerically solve magneto-hydrodynamics (MHD) flow problems. MHD flow equations describe the motion of electrically conducting fluids in the presence of magnetic fields. We study the flow of an incompressible, viscous and electrically conducting fluid in a duct with partially conducting and partially non-conducting walls under the influence of a uniform magnetic field. The magnetic field influences the fluid motion and, in turn, the fluid motion changes the magnetic field.

In 1937 Hartmann [1] pioneered the investigation of MHD flow for viscous, incompressible, electrically conducting fluid between two parallel plates in the presence of a transverse magnetic field. Since then, many researchers have been studying MHD flows through channels (ducts), mainly, due to their numerous applications in pumps, accelerators, flow-meters, astrophysics, geology, power generation, thermonuclear reactor technology, designing cooling 
systems with liquid metals, MHD generators, medicine, etc. The flow parameters that characterize the dynamical regime of MHD are Reynolds $\left(R e=U L / v_{f}\right.$, where $U$ is a characteristic velocity, $L$ is a characteristic length and, $v_{f}$ is the kinematic viscosity of the fluid) and Hartmann $(H a)$ number $\left(H a=L B_{0} \sqrt{\frac{\sigma}{\rho v_{f}}}\right.$, where $B_{0}$ is the imposed magnetic field strength, $\rho$ is the mass density of the fluid and, $\sigma$ is the conductivity of the fluid). Hartmann number measures the effects of the magnetic field; strong magnetic fields $(H a>>1)$ tend to make the flow uniform (quasi-2D) along the direction of the field.

Following the work of Hartmann [1], a number of theoretical and numerical studies focused on laminar, pressure-driven MHD flow in straight channels and ducts subjected to various electrical boundary conditions [2-5]. For a limited number of MHD flow cases, involving noncomplex geometry (e.g. circular, rectangular or elliptical cross sections) and simple boundary conditions, analytical solutions can be obtained [6, 2]. In [4], Hunt and Stewartson applied a boundary-layer technique to study flow in a duct with electrically conducting walls perpendicular and parallel to magnetic field $\boldsymbol{B}$ and, derived expressions for the flow rate in the form of an asymptotic expansion in the limit of large Hartmann number (neglecting secondary flows). In [7], Baylis and Hunt perform a comprehensive flow study for a cylindrical annulus with rectangular cross section, by considering the walls perpendicular to the magnetic field electrically insulated and those parallel electrically conductive. In [8], Tabeling and Chabreri present a boundary-layer study on the secondary laminar MHD flow formed in the high Hartmann number $M$ regime, by considering annular ducts with rectangular cross section.

In the majority of the MHD flow problems considered, the flow equations can only be solved numerically and, therefore, several numerical methods have been used, such as Finite Difference Method (FDM), Finite Element Method (FEM), the closely related Finite Volume Method (FVM) and Boundary Element Method (BEM). The well-established numerical method like FDM [9, 10], FEM [11-15] and BEM [16-20] applied to MHD flow problems for low $(M \leq 100)$, moderate $(100 \leq M \leq 1000)$ and high $(M \geq 1000)$ Hartmann numbers $(M$ is used as the squared Hartman number $M=H a^{2}$ ). Due to the drawbacks of the mesh-based numerical methods [21], meshless methods (MM) were introduced in the 1970's and have been intensively developed over the past two decades [21]. MM emerged as a potential alternative to traditional mesh-based numerical methods, alleviating the burden of mesh generation. MM approximate the unknown field functions and their derivatives based on a set of nodes distributed over the spatial domain. 
A variety of meshless methods have been proposed since the first one, Smoothed Particle Hydrodynamics (SPH), was introduced [22], including: the Diffuse Approximation Method (DAM) [23], which is closely related to the Moving Least Squares (MLS) approximation method; the Element Free Galerkin method (EFG) [24]; the Reproducing Kernel Particle Method (RKPM) [25, 26], which has been used to improve the SPH approximation; the Partition of Unity Finite Element Method (PUFEM) [27]; the $h p$-Clouds method [28]; the Moving Least-Square Reproducing Kernel Method (MLSRK) [29]; the meshless Local Boundary Integral Equation method (LBIE) [30]; the Meshless Local Petrov-Galerkin method (MLPG) [31, 32]; the Finite Point method (FPM) [33]; the Meshless Point Collocation method (MPC) [34]; the Method of Fundamental Solutions (MFS) [35] and the Method of Particular Solutions (MPS) [36]. Despite that meshless methods haven been mainly used for solving hydrodynamics problems [21], studies which focus on MHD problems are limited. Smoothed particle hydrodynamics (SPH) [37, 38], Element-Free Galerkin (EFG) method [39], meshless point collocation method (MPCM) [40, 41], local boundary integral equation (LBIE) meshless method [42], Meshless Local Petrov-Galerkin (MLPG) method [43], the meshfree weak-strong method (MWS) [44] and the radial basis function (RBF) [45, 46] have been successfully used to solve MHD flow problems.

The present paper reports on the application of the Meshless Point Collocation (MPC) method for solving transient MHD flow in a rectangular duct. The governing equations are solved numerically by using the explicit Euler integration scheme for temporal discretization and the Discretization Corrected Particle Strength Exchange (DC PSE) method for the spatial interpolation of the field variables (spatial derivatives up to second order). To the authors' knowledge, no previous work has combined MPC and DC PSE for solving the electrically driven MHD flow with high Hartmann numbers. We solve the strong-form governing equations by using the collocation method and we apply efficient local refinement strategies $[47,48]$ to resolve boundary layers that occur at high Hartmann numbers. We use uniform and irregular nodal distributions to verify the accuracy and the reproducibility of the proposed scheme. As pointed out in [34], uniform nodal distributions typically exhibit higher accuracy than irregular nodal distributions of similar nodal density. In MHD flows cases, the forming boundary layers, especially for higher values of the Hartmann number, can be accurately resolved by using locally refined nodal distributions. In this paper, we show that irregular nodal distributions can be as accurate as uniform ones when using DC PSE and local nodal refinement. Furthermore, we apply an efficient method to impose Neumann boundary conditions, by using interior nodes in the support domain of the Neumann boundary nodes. 
The enforcement of boundary conditions (Dirichlet and Neumann) is important issue for the numerical methods used in the solution of non-stationary partial differential equations (PDEs), as it affects both the accuracy and the stability of the time integration algorithm. Efficient enforcement of Neumann boundary conditions makes explicit solvers a suitable alternative to implicit ones, leading to less computational cost and a code that is inherently parallelizable.

The outline of the paper is as follows. In Section 2, we present the governing MHD equations along with the corresponding boundary and initial conditions. In section 3 , we give an overview of the approximation scheme, Discretization Corrected Particle Strength Exchange (DC PSE), while in section 4 we present the time discretization scheme (explicit solver), the imposition of Neumann boundary conditions and a stability analysis of the explicit solver used. Section 5 includes several numerical examples depicting the accuracy and efficiency of the proposed scheme. Finally, conclusions are presented in Section 6.

\section{Equations and Boundary Conditions}

We consider the non-stationary, laminar flow of a viscous, incompressible and electrically conducting fluid in a rectangular duct, driven by electrodes connected to external circuits, as shown in Fig.1.

[Figure 1 about here]

Fig. 1. Geometry and boundary conditions for the MHD flow problem.

The longitudinal axis of the duct is chosen as the $z$-direction. A uniform magnetic field of intensity $\boldsymbol{B}_{0}$ is imposed along a random vector having an angle $\varphi$ with the $y$-axis. The fluid flow is considered to be fully developed (the duct is of infinite length) and the walls are considered either electrically insulated or having variable conductivity.

The basic equations governing the MHD duct flow are obtained using Maxwell's equations for the electromagnetic field, Ohm's law, equation of continuity and the Navier-Stokes equations [49]. There is only one velocity component $V_{z}(x, y, t)$ and one component $B_{z}(x, y, t)$ of the induced magnetic field (in the $z$-direction). All physical quantities except pressure are independent of the spatial variable $z$ and, therefore, the magnetic vector takes the form $\boldsymbol{B}=\left(B_{0}\right.$, $\left.0, B_{z}(x, y, t)\right)$. Additionally, we assume that displacement currents are negligible. The partial equations in non-dimensional form, in terms of velocity $V(x, y, t)$ and induced magnetic field $B(x, y, t)$, become 


$$
\begin{aligned}
& \frac{\partial V}{\partial t}=\nabla^{2} V+M_{x} \frac{\partial B}{\partial x}+M_{y} \frac{\partial B}{\partial y} \\
& \frac{\partial B}{\partial t}=\nabla^{2} B+M_{x} \frac{\partial V}{\partial x}+M_{y} \frac{\partial V}{\partial y}
\end{aligned}
$$

where $M_{x}=M \sin \varphi, M_{y}=H a \cos \varphi$ and $M=\frac{B_{0}^{2} L^{2} \sigma}{v_{f}}$ is the squared Hartmann number. The boundary conditions are defined as

$$
\begin{gathered}
V=0 \text { at } y= \pm 1 \text { and } x= \pm 1 \\
B=k \text { for } x>l, y= \pm 1 \text { and } x=1 \\
B=-k \text { for } x<-l, y= \pm 1 \text { and } x=-1 \\
\frac{\partial B}{\partial y}=0 \text { for }-l \leq x \leq l, y= \pm 1
\end{gathered}
$$

where $k$ (wall inductance) is a constant $(k \leq 1)$ and $l$ defines the size of the electrodes. The initial conditions are:

$$
\begin{aligned}
& V(x, y, 0)=0 \\
& B(x, y, 0)=0
\end{aligned}
$$

\section{Discretization Corrected Particle Strength Exchange (DC PSE) method}

DC PSE is a Lagrangian particle based solution method introduced in [50]. To solve PDEs using DC PSE meshless method, authors in [51] have reformulated DC PSE method to work in the Eulerian framework. The DC PSE method is based on Particle Strength Exchange (PSE) operators. Following, we present the PSE operators and the DC PSE interpolation method.

\subsection{Particle Strength Exchange (PSE) operators}

The Particle Strength Exchange (PSE) method makes use of kernels to create approximate differential operators that guarantee the conservation of particle strength in particle-particle interactions. It was first introduced in 1989 by Degond and Mas-Gallic [52] for diffusion and convection-diffusion problems. Based on this initial work, Eldredge et al. [53] developed a framework for approximating arbitrary derivatives. In general, a PSE operator $Q^{\boldsymbol{\beta}} f(\boldsymbol{x})$ for approximating the derivative $D^{\boldsymbol{\beta}} f(\boldsymbol{x})$ has the form

$$
Q^{\boldsymbol{\beta}} f(\boldsymbol{x})=\frac{1}{\varepsilon^{|\boldsymbol{\beta}|}} \int(f(\boldsymbol{y}) \mp f(\boldsymbol{x})) \eta_{\varepsilon}^{\boldsymbol{\beta}}(\boldsymbol{x}-\boldsymbol{y}) d \boldsymbol{y}
$$


with $\eta_{\varepsilon}^{\boldsymbol{\beta}}=\eta^{\boldsymbol{\beta}}(x / \varepsilon) / \varepsilon^{d}$ being a scaled kernel of radius $\varepsilon(\varepsilon=\boldsymbol{h}$, where $\boldsymbol{h}$ is the nodal spacing) and, $d$ being the number of dimensions. For conservation reasons, the sign in Eq. (9) is negative when $|\boldsymbol{\beta}|$ is even and positive when $|\boldsymbol{\beta}|$ is odd, with $\boldsymbol{\beta}$ a multi-index [50]. The challenge is to find a kernel $\eta_{\varepsilon}^{\beta}$ that leads to accurate approximations for $D^{\beta}$. To find such kernels for arbitrary derivatives, the idea in [50] is adopted, starting from the Taylor expansion of a function $f(\boldsymbol{y})$ about a point $\boldsymbol{x}$ :

$$
f(\boldsymbol{y})=f(\boldsymbol{x})+\sum_{|\boldsymbol{a}|=1}^{\infty} \frac{1}{\boldsymbol{a} !}(\boldsymbol{y}-\boldsymbol{x})^{\boldsymbol{a}} D^{\boldsymbol{a}} f(\boldsymbol{x})
$$

The next step is to subtract or add $f(\boldsymbol{x})$, depending on $|\boldsymbol{\beta}|$ being odd or even, on both sides and convolute the equation with the unknown kernel $\eta_{\varepsilon}^{\boldsymbol{\beta}}$. This lead, by taking into account Eq. (9), to

$$
Q^{\boldsymbol{\beta}} f(\boldsymbol{x})=\frac{1}{\varepsilon^{|\boldsymbol{\beta}|}} \sum_{|\boldsymbol{a}|=1}^{\infty} \frac{1}{\boldsymbol{a} !} D^{\boldsymbol{a}} f(\boldsymbol{x}) \int(\boldsymbol{y}-\boldsymbol{x})^{\boldsymbol{a}} \eta_{\varepsilon}^{\boldsymbol{\beta}}(\boldsymbol{x}-\boldsymbol{y}) d \boldsymbol{y}
$$

By introducing the continuous $\boldsymbol{\alpha}$-moments

$$
M_{a}=\int(x-y)^{\alpha} \eta^{\beta}(x-y) d y=\int z^{\alpha} \eta^{\beta}(z) d z
$$

and isolating the derivatives $D^{\boldsymbol{\beta}}$ on the right-hand side of Eq. (11) we obtain:

$$
Q^{\boldsymbol{\beta}} f(\boldsymbol{x})=\frac{(-1)^{|\boldsymbol{\beta}|}}{\boldsymbol{\beta} !} M_{\boldsymbol{\beta}} D^{\boldsymbol{\beta}} f(\boldsymbol{x})+\sum_{\substack{|\boldsymbol{a}|=1 \\ \boldsymbol{a} \neq \boldsymbol{\beta}}}^{\infty} \frac{(-1)^{|\boldsymbol{a}|}}{\boldsymbol{a} !} \varepsilon^{|\boldsymbol{a}|-|\boldsymbol{\beta}|} M_{\boldsymbol{a}} D^{\boldsymbol{a}} f(\boldsymbol{x})
$$

Finally, to approximate $Q^{\beta} f(\boldsymbol{x})$ with order of accuracy $r$ (in the present study we apply second order accuracy $(\boldsymbol{r}=\mathbf{2})$ ), the following set of conditions is imposed for the moments $M_{a}$ :

$$
M_{\boldsymbol{a}}=\left\{\begin{array}{cc}
(-1)^{|\boldsymbol{\beta}|} \boldsymbol{\beta} ! & \boldsymbol{a}=\boldsymbol{\beta} \\
0, & \boldsymbol{a} \neq \boldsymbol{\beta}, \quad 1 \leq|\boldsymbol{a}| \leq|\boldsymbol{\beta}|+r-1
\end{array}\right.
$$

In addition, if we impose

$$
\int|z|^{|\boldsymbol{\beta}|+r}\left|\eta^{\boldsymbol{\beta}}(\mathbf{z})\right| d \mathbf{z}<\infty
$$

the mollification error $\epsilon_{\varepsilon}(\boldsymbol{x})=D^{\boldsymbol{\beta}} f(\boldsymbol{x})-Q^{\boldsymbol{\beta}} f(\boldsymbol{x})$ is bounded [53] 
The challenge is to construct a kernel that satisfies the conditions described in Eq. (14). A possible procedure has been described in [53]. Once the kernel is defined, the operator in Eq. (9) can be discretised using a midpoint quadrature over the nodes as

$$
Q_{h}^{\boldsymbol{\beta}} f(\boldsymbol{x})=\frac{1}{\varepsilon^{|\boldsymbol{\beta}|}} \sum_{p \in N(\boldsymbol{x})}\left(f\left(\boldsymbol{x}_{p}\right) \mp f(\boldsymbol{x})\right) \eta_{\varepsilon}^{\boldsymbol{\beta}}\left(\boldsymbol{x}-\boldsymbol{x}_{p}\right) V_{p}
$$

where $N(\boldsymbol{x})$ is the number of all nodes in a neighborhood around $\boldsymbol{x}$, which is usually defined by a cutoff radius $r_{c}$, usually chosen such that $N(\boldsymbol{x})$ coincides to a certain level of accuracy with the kernel support, and $V_{p}$ which is the volume associated with each particle. Given such a discretization, the discretization error $\epsilon_{h}(\boldsymbol{x})=Q^{\boldsymbol{\beta}} f(\boldsymbol{x})$ - $Q_{h}^{\boldsymbol{\beta}} f(\boldsymbol{x})$ is also bounded [53].

\subsection{The Discretization Corrected PSE operators}

The Discretization Corrected PSE (DC PSE) operators were introduced in [50], to reduce the discretization error $\epsilon_{h}(x)$ in the PSE operator approximation. The derivation of the DC PSE approximation starts from Eq. (16) (with $V_{p}$ included in the kernel function), with the requirement of finding a kernel function which minimizes the difference between this specific discrete operator and the actual derivative. To achieve this, each term $f\left(\boldsymbol{x}_{p}\right)$ in Eq. (16) is replaced with its Taylor expansion about $\boldsymbol{x}$, leading to the following expression for the derivative approximation:

$$
Q_{h}^{\boldsymbol{\beta}} f(\boldsymbol{x})=\frac{(-1)^{|\boldsymbol{\beta}|}}{\boldsymbol{\beta} !} Z_{h}^{\boldsymbol{\beta}} D^{\boldsymbol{\beta}} f(\boldsymbol{x})+\sum_{\substack{|\boldsymbol{a}|=1 \\ \boldsymbol{a} \neq \boldsymbol{\beta}}}^{\infty} \frac{(-1)^{|\boldsymbol{a}|}}{\boldsymbol{a} !} \varepsilon^{|\boldsymbol{a}|-|\boldsymbol{\beta}|} Z_{h}^{\boldsymbol{a}} D^{\boldsymbol{a}} f(\boldsymbol{x})+r_{0}
$$

with

$$
r_{0}=\left\{\begin{array}{cc}
0, & |\boldsymbol{\beta}| \text { even } \\
2 e^{-|\boldsymbol{\beta}|} Z_{h}^{0} f(\boldsymbol{x}) & |\boldsymbol{\beta}| \text { odd }
\end{array}\right.
$$

and the discrete moments defined as

$$
Z_{h}^{a}=\frac{1}{\varepsilon^{d}} \sum_{p \in N(x)}\left(\frac{x-x_{p}}{\varepsilon}\right)^{a} \eta^{\beta}\left(\frac{x-x_{p}}{\varepsilon}\right)
$$


Therefore, the set of moment conditions become

$$
Z_{h}^{a}=\left\{\begin{array}{cc}
(-1)^{|\boldsymbol{\beta}|} \boldsymbol{\beta} ! & \boldsymbol{a}=\boldsymbol{\beta} \\
0 & \boldsymbol{a} \neq \boldsymbol{\beta} \\
<\infty & \text { otherwise }
\end{array} \quad a_{\min } \leq|\boldsymbol{a}| \leq|\boldsymbol{\beta}|+r-1\right.
$$

for all $|\boldsymbol{\beta}| \neq 0$, where $a_{\text {min }}$ is one when $|\boldsymbol{\beta}|$ is odd and 0 when $|\boldsymbol{\beta}|$ is even. The kernel $\eta^{\boldsymbol{\beta}}$ is chosen as:

$$
\eta^{\boldsymbol{\beta}}(\boldsymbol{x}, \mathbf{z})=\left(\sum_{|\gamma|=a_{\min }}^{|\boldsymbol{\beta}|+r-1} a_{\gamma}(\boldsymbol{x}) \mathbf{z}^{\gamma}\right) e^{-|\mathbf{z}|^{2}}=P(\boldsymbol{x}, \boldsymbol{z}) W(\mathbf{z}), \quad \boldsymbol{z}=\frac{\boldsymbol{x}-\boldsymbol{x}_{p}}{\varepsilon}
$$

The kernel function consists of a polynomial correction function $P(\boldsymbol{x}, \boldsymbol{z})$ and the weight function $W(\mathbf{z})$. Different choices for the weight function applied can be found in [54].

The unknown coefficients $a_{\boldsymbol{\gamma}}(\boldsymbol{x})$ are obtained by requesting the kernel given by Eq. (21) to satisfy the conditions (20), resulting in the following linear system of equations:

$$
\sum_{|\gamma|=a_{\text {min }}}^{|\boldsymbol{\beta}|+r-1} a_{\gamma}(\boldsymbol{x}) w_{\boldsymbol{a}}^{\gamma}(\boldsymbol{x})=\left\{\begin{array}{cc}
(-1)^{|\boldsymbol{\beta}|} \boldsymbol{\beta} ! & \boldsymbol{a}=\boldsymbol{\beta} \\
0 & \boldsymbol{a} \neq \boldsymbol{\beta}
\end{array} \quad, \forall a_{\text {min }} \leq|\boldsymbol{a}| \leq|\boldsymbol{\beta}|+r-1\right.
$$

with weights

$$
w_{\boldsymbol{a}}^{\gamma}(\boldsymbol{x})=\frac{1}{\varepsilon^{|\boldsymbol{a}+\gamma|+d}} \sum_{p \in N(\boldsymbol{x})}\left(\boldsymbol{x}-\boldsymbol{x}_{p}\right)^{\boldsymbol{a}+\gamma} e^{-\left(\frac{\left|x-x_{p}\right|}{\varepsilon}\right)^{2}}
$$

To compute the approximated derivative $Q_{h}^{\boldsymbol{\beta}} f(\boldsymbol{x})$ at node $\boldsymbol{x}_{p}$, the coefficients are found by solving the above linear system of equations for $\boldsymbol{x}=\boldsymbol{x}_{p}$. Given our choice of kernel function, the DC PSE derivative approximation becomes:

$$
Q_{h}^{\boldsymbol{\beta}} f\left(\boldsymbol{x}_{p}\right)=\frac{1}{\varepsilon\left(\boldsymbol{x}_{p}\right)^{\boldsymbol{\beta}}} \sum_{\boldsymbol{x}_{q} \in \mathcal{N}\left(x_{p}\right)}\left(f\left(\boldsymbol{x}_{q}\right) \mp f\left(\boldsymbol{x}_{p}\right)\right) \boldsymbol{p}\left(\frac{\boldsymbol{x}-\boldsymbol{x}_{p}}{\varepsilon\left(\boldsymbol{x}_{p}\right)}\right) \boldsymbol{a}^{T}\left(\boldsymbol{x}_{p}\right) e^{-\left(\frac{\left|\boldsymbol{x}_{p}-\boldsymbol{x}_{q}\right|}{\varepsilon\left(\boldsymbol{x}_{p}\right)}\right)^{2}}
$$

where $\boldsymbol{p}(\boldsymbol{x})=\left[p_{1}(\boldsymbol{x}), p_{2}(\boldsymbol{x}), \ldots, p_{m}(\boldsymbol{x})\right]$, with $m$ being the number of monomial used $(m=6$ and 10 for second order monomials in two and three dimensions, respectively) and, $\boldsymbol{a}(\boldsymbol{x})$ are the vectors of terms in the monomial basis and their coefficients, respectively. 


\section{Solution procedure}

\subsection{Explicit solver: Euler scheme}

We solve the MHD flow equations (1) and (2) by using an explicit time integration method, while spatial derivatives are computed using the Discretization Corrected Particle Strength Exchange interpolation method presented in the previous section.

By using Euler's explicit time-stepping scheme, the MHD flow equations become:

$$
\begin{aligned}
& \frac{V^{(n+1)}-V^{(n)}}{\delta t}=\nabla^{2} V^{(n)}+M_{x} \frac{\partial B^{(n)}}{\partial x}+M_{y} \frac{\partial B^{(n)}}{\partial y} \\
& \frac{B^{(n+1)}-B^{(n)}}{\delta t}=\nabla^{2} B^{(n)}+M_{x} \frac{\partial V^{(n)}}{\partial x}+M_{y} \frac{\partial V^{(n)}}{\partial y}
\end{aligned}
$$

where $(n)$ is the time step index and $\delta t$ is the time step. After computing the updated values $V^{(n+1)}$ and $B^{(n+1)}$ we apply the boundary conditions; we can impose the Dirichlet boundary conditions directly, since we are using a strong form solution method (MPC), while Neumann boundary conditions need special treatment, as will be explained in detail in section 4.2.

When using an explicit time integration scheme there is no need to solve a linear system of equations; instead, the field values at the next time step are computed based on the values from the previous step $\left(V^{(n+1)}\right.$ and $B^{(n+1)}$ are computed based on the $V^{(n)}$ and $\left.B^{(n)}\right)$. Compared to implicit solvers, this is a significant advantage in terms of code implementation, storage and computation cost. Nevertheless, explicit solvers are only conditionally stable, with the maximum time step that can be used limited by the Courant-Friedrichs-Levy (CFL) stability criteria (details in section 4.3).

\subsection{No flux boundary conditions}

For many PDE problems, the governing equations include Neumann boundary conditions (no flux boundary conditions) of the form

$$
\boldsymbol{\nabla} q \cdot \boldsymbol{n}=0
$$

where $\boldsymbol{n}$ is a unit normal vector and $q$ is the unknown scalar field function ( $V$ or $B$ in our case). We enforce such Neumann boundary conditions by including in the support domain of the 
Neumann boundary only interior nodes when computing the derivatives in Eq. (27), as shown in Fig. 2.

[Figure 2 about here]

Fig. 2. The support domain used for enforcing no flux boundary conditions for a boundary node. The boundary node is marked with a circle (red) and the nodes in its support domain with a square (green).

This choice of support domain decouples the Neumann boundary conditions equations, allowing direct computation of the values on the boundary based on known interior values. For example, let's consider a two-dimensional problem with normal vector $\boldsymbol{n}$ given as $\boldsymbol{n}=\left(n^{x}, n^{y}\right)$. The derivatives that appear in the Neumann boundary conditions equations are computed using the DC PSE approximation using Eq. (24), leading to the following expression for a boundary node $p$ :

$$
\frac{\partial q_{p}}{\partial x}=\sum_{\boldsymbol{x}_{i} \in \mathcal{N}\left(\boldsymbol{x}_{p}\right)} w_{i}^{x} q_{i} \quad \frac{\partial q_{p}}{\partial y}=\sum_{\boldsymbol{x}_{i} \in \mathcal{N}\left(\boldsymbol{x}_{p}\right)} w_{i}^{y} q_{i}
$$

With $w_{i}^{x}$ and $w_{i}^{y}$ being the weights of the first order spatial derivatives and therefore, the Neumann boundary condition for node $p$ becomes:

$$
\sum_{\boldsymbol{x}_{i} \in \mathcal{N}\left(x_{p}\right)} w_{i}^{x} q_{i} n^{x}+\sum_{\boldsymbol{x}_{i} \in \mathcal{N}\left(x_{p}\right)} w_{i}^{y} q_{i} n^{y}=0
$$

allowing the computation of the value that has to be imposed at the boundary node as:

$$
q_{p}=\frac{-1}{n_{p}^{x} w_{p}^{x}+n_{p}^{y} w_{p}^{y}}\left(\sum_{\boldsymbol{x}_{i} \in \mathcal{N}\left(\boldsymbol{x}_{p}\right), i \neq p} w_{i}^{x} q_{i} n^{x}+\sum_{\boldsymbol{x}_{i} \in \mathcal{N}\left(\boldsymbol{x}_{p}\right), i \neq p} w_{i}^{y} q_{i} n^{y}\right)
$$

\subsection{Stability conditions for explicit time integration}

To compute the critical time step needed to obtain stable results, we rewrite Eqs. (25)-(26) as

$$
\left[\begin{array}{l}
V^{(n+1)} \\
B^{(n+1)}
\end{array}\right]=\left[\begin{array}{l}
V^{(n)} \\
B^{(n)}
\end{array}\right]+\delta t\left[\begin{array}{cc}
\nabla^{2} V^{(n)} & M_{x} \frac{\partial B^{(n)}}{\partial x}+M_{y} \frac{\partial B^{(n)}}{\partial y} \\
M_{x} \frac{\partial V^{(n)}}{\partial x}+M_{y} \frac{\partial V^{(n)}}{\partial y} & \nabla^{2} B^{(n)}
\end{array}\right]
$$

which can be written in matrix form as:

$$
\mathbf{U}^{(n+1)}=[\mathbf{I}+\delta t \mathbf{A}] \mathbf{U}^{(n)}
$$


where $\mathbf{U}^{(n+1)}=\left[\begin{array}{l}V^{(n+1)} \\ B^{(n+1)}\end{array}\right]$ and $\mathbf{A}=\left[\begin{array}{ll}\mathbf{L} & \mathbf{K} \\ \mathbf{K} & \mathbf{L}\end{array}\right]$, with the matrices $\mathbf{K}$ and $\mathbf{L}$ representing the following linear operators approximated using DC PSE:

$$
\begin{gathered}
\mathbf{L}=\frac{\partial^{2}}{\partial x^{2}}+\frac{\partial^{2}}{\partial y^{2}} \\
\mathbf{K}=M_{x} \frac{\partial}{\partial x}+M_{y} \frac{\partial}{\partial y}
\end{gathered}
$$

The explicit method defined by Eq. (32) is stable if

$$
\left|1+\delta t \lambda_{A}\right| \leq 1
$$

where $\lambda_{A}$ are the eigenvalues of the matrix $\mathbf{A}$. The above stability condition requires the eigenvalues $\lambda_{A}$ to be either real and negative, leading to

$$
\delta t \leq \frac{2}{\left|\lambda_{A}\right|}
$$

or complex with negative real parts, leading to

$$
\delta t \leq \frac{-2 \operatorname{Re}\left(\lambda_{A}\right)}{\left|\lambda_{A}\right|^{2}} .
$$

The stability criteria above involve the eigenvalues of matrix A. When the problem discretisation includes a large number of nodes, matrix $\mathbf{A}$ becomes very large, and calculating its eigenvalues is not practical. We note the fact that the diagonal terms of matrix $\mathbf{A}$ are determined by the Laplacian operator $\mathbf{L}$, consisting of second order derivatives, while the off-diagonal terms defined by operator $\mathbf{K}$ includes only first order derivatives. This implies that the relative weight of the two operators on the structure of matrix $\mathbf{A}$ is dictated by discretisation: a more refined discretisation leads to a higher weight of operator $\mathbf{L}$ and lower influence of operator $\mathbf{K}$. If the discretisation is not refined enough, the higher weight of offdiagonal operator $\mathbf{K}$ can lead to eigenvalues with a positive real part; in such case explicit time integration is not possible and discretisation has to be refined. A positive aspect of a refined discretisation is that the resulting matrix is diagonally dominant, having its eigenvalues distributed close to the real axis. Therefore, we can estimate the maximum time step using Eq. (36), with an upper bound of the maximum eigenvalue of matrix A computed using the Greshgorin circle theorem [55]. Given the composition of matrix A, the upper bound of the maximum eigenvalue can be computed without assembling the matrix:

$$
\left|\lambda_{A}\right| \leq \max _{i}\left(\sum_{j}\left(\left|L_{i j}\right|+\left|K_{i j}\right|\right)\right)
$$


If the time step for explicit integration is not appropriate (too long), the algorithm will diverge rapidly. In such case, the time discretization is refined and then time integration is performed again.

\section{Results}

In this section, we present the results computed using the proposed numerical scheme. We consider different values for the wall inductance $k$, Hartmann number $M$ and electrode length $l$, and examine how they influence the velocity $V$ and the induced magnetic field $B$.

For high Hartmann numbers boundary layers are formed. To capture the steep gradients and to eliminate unwanted numerical oscillations we apply local refinement. Several refinement strategies have been proposed, such as nodal refinement, enlargement of the local support domain, fully upwind support domain and adaptive upwind support domain [56]. By enlarging the local support domain, the upstream information can be captured; however, the accuracy of the solution might be reduced [56], especially in regions with high field gradients. When using an upwind support domain, the accuracy and stability of the solution are improved for high Hartmann numbers. Nevertheless, these methods give very poor results for low and moderate Hartmann numbers.

In the present study, we applied an $h$-type refinement, by increasing the number of nodes close to the boundaries. The spatial domain is discretized by a set of nodes distributed either uniformly or randomly in space.

\subsection{Proposed method verification}

We verify the accuracy of the proposed solution method by computing the flow field of a viscous, incompressible and electrically conducting fluid [40] in a rectangular duct with square cross section, defined over the domain $(-1,1) \times(-1,1)$ as shown in Fig. 3. The fluid is subjected to a magnetic field in the $y$-direction (i.e. $\varphi=\frac{\pi}{2}$ ) having insulating walls $(B=0)$ and no-slip boundary velocity conditions $(V=0)$.

[Figure 3 about here]

Fig. 3. Geometry and boundary conditions for the MHD flow in a duct with square cross section and insulating walls. 
We compare the numerical findings obtained using the proposed solution method with those presented in the literature [57, 40] for Hartmann numbers $M=100, M=500$ and $M=1,000$. The maximum stable time step for numerical integration is computed using the Greshgorin circle theorem presented above. We use uniform and irregularly distributed sets of nodes to represent the spatial domain. We use successively denser nodal distributions to get a grid independent numerical solution. For $M=100$ we consider five different nodal distributions with increasing nodal density, consisting of $81 \times 81,161 \times 161,321 \times 321,641 \times 641$ and $1281 \times 1281$ nodes, respectively. We apply global refinement since for low Hartmann numbers the boundary layers are not very pronounced. We compute the spatial derivatives of the unknown field functions (velocity and magnetic field) with an in-house $\mathrm{C}++$ code. The computational time (in seconds) for the derivatives (up to second order) was $84 \mathrm{msec}, 332 \mathrm{msec}, 1.37 \mathrm{sec}, 5 \mathrm{sec}$ and $23 \mathrm{sec}$, respectively. Table 1, lists the values for the critical time step computed through the Gerschgorin theorem, for different values of $M(M=100,500$ and 1,000) and nodal distributions.

[Table 1 about here]

Table 1. Critical time step for increasingly denser nodal distributions and for Hartmann number $\mathrm{M}=100,500$ and 1000.

For higher Hartmann numbers, local refinement is used in both uniform and irregular node distribution, as it shown in Fig. 4. Local refinement allows us to capture the boundary layers without the need for global refinement, drastically reducing the computational cost.

Table 2(a-b) presents the analytical and numerical findings at specific sites in the spatial domain for Hartmann numbers $M=100$ and $M=500$. Table $2 \mathrm{c}$ shows the values of the velocity field, compute at specified sites, with successively denser nodal distributions. It can be seen that as the nodal distribution increases the values convergence to the analytical solution. The numerical results are compared against the analytical solution at steady state [57] and finite element method [40].

[Table 2 about here]

Table 2. 
[Figure 4 about here]

Fig. 4. Locally refined (a) uniform and (b) irregular nodal distributions.

Fig. 5 shows the contour plots for the velocity and magnetic field for Hartmann numbers $M=100,500$ and 1,000. We observe that the boundary layers are resolved accurately and no oscillations in the numerical solution are present.

[Figure 5 about here]

Fig. 5. Contour plots of the velocity (left) and induced magnetic field (right) for Hartmann numbers (a) $M=100$ (b) $M=500$ and (c) $M=1000$.

5.2 Illustration of the proposed algorithm performance: MHD flow in a rectangular duct with insulating walls

The following test cases refer to MHD flow in a rectangular duct with insulating walls. For all the examples examined, we report on the computational efficiency (critical time step, time (in sec) to compute spatial derivatives) and the accuracy of the proposed scheme.

\subsubsection{Hartmann number influence}

We examine the laminar, incompressible and electrically conducting fluid flow in a long duct of square cross-section defined over the domain $(-1,1) \times(-1,1)$. We consider varying wall conductivity, having the applied magnetic field directed along the $y$-axis. Flow is driven downwards by the electrodes, which are placed in the middle of the top and bottom walls, perpendicular to the applied magnetic field, as shown in Fig. 1. The walls parallel to the applied magnetic field are kept at constant inductance, yet opposite in sign. On the insulated walls, identical magnetic field values are continued from the parallel walls. The external magnetic field applied results in an induced current within the fluid, forcing it to flow in the external circuits. We solve the dynamic flow problem until reaching the steady-state solution $(\tau \rightarrow \infty$ for the time domain $(0, \tau))$. For the numerical simulation, we use different values of Hartmann number, while setting wall inductance $k=1$, electrode length $l=0.3$ and angle $\varphi=\pi / 2$. 
We use an irregular nodal distribution set of 435,014 nodes in total (like in Fig. 4b), locally refined close to the boundaries and we plot the numerical results when steady state is reached. This locally refined grid can capture the boundary layers formed at $x=1$ and $x=-1$ for Hartmann numbers up to $M=2,000$.

[Figure 6 about here]

Fig. 6. Contour plots of the velocity field (left) and the induced magnetic field (right) for Hartmann numbers (a) $M=1,000$ and (b) $M=2,000$.

Fig. 6 shows the contour plots for the velocity field and the induced magnetic field for Hartmann numbers $M=1,000$ and 2,000. The numerical results obtained are compared against those presented in [58] (in [58] authors used the finite element method to solve the flow equations). The numerical results are in a very good agreement for $M$ up to $M=1,000$, without any spurious oscillations being present. Furthermore, in the present study we computed results for higher $M$ numbers $(M=2,000)$. In the present scheme, local refinement used eliminates the spurious oscillations. The values of the velocity and magnetic field at prescribed locations and at different times are listed in Table 3. As the Hartmann number increases, flow velocity becomes uniform throughout the region, while at a very narrow part of the domain near the walls, boundary layers are formed. A boundary layer is also observed for the induced magnetic field, starting at the points of discontinuity $(l, \pm 1)$ on the boundary.

[Table 2 about here]

Table 3. Flow field values for Hartmann numbers $M=1,000$ and 2,000 at different time instances.

\subsubsection{Impact of $l, k$ and $\varphi$ parameters on flow regime}

We examine how the flow regime is influenced by the wall inductance $k$, electrode length $l$ and magnetic field imposition angle $\varphi$ using the contour plots of the velocity and the induced magnetic fields. We consider an irregular nodal distribution, locally refined close to the boundaries, as in the previous section.

Fig. 7 shows the effect of the electrode length $l$ on the flow velocity $V$ and the induced magnetic field $B$ for Hartmann number $M=500$. 
[Figure 7 about here]

Fig. 7. Contour plots of the velocity field and the induced magnetic field for Hartmann number $M=500$, wall inductance $k=1$, magnetic field imposition angle $\varphi=\pi / 2$ and electrode

$$
\text { length (a) } l=0.3 \text { (b) } l=0.5 \text { and (c) } l=0.7 \text {. }
$$

For both velocity $V$ and magnetic field $B$, the boundary layers become less pronounced when the electrode length $l$ increases. As $l$ increases, the boundary layers in the induced magnetic field are confined to the walls $x= \pm 1$, being combined with the layers emerging from the insulated walls. An increase in the length $l$ results in an enlargement of the stagnant region of the magnetic field in front of the conducting portions of the boundary.

Fig. 8 shows the behavior of the velocity and the induced magnetic field for different values of the wall inductance $k$ for Hartmann number $M=500$.

[Figure 8 about here]

Fig. 8. Contour plots of the velocity field (left) and the induced magnetic field (right) for Hartmann number $M=500$, electrode length $l=0.3$, magnetic field imposition angle $\varphi=\pi / 2$ and wall inductance (a) $k=1$ (b) $k=-1$ and (c) $k=0.3$.

As wall inductance $k$ increases, the velocity and the induced magnetic field values increase. This indicates the importance of the wall inductance when high velocity and induced magnetic field are required in the direction of the flow. Additionally, it can be observed that the velocity filed changes direction in the duct and the current lines change the direction in the left and right half of the duct when $k$ changes sign. Therefore, it is possible to control the direction of the flow.

Finally, we examine the flow regime when the induced magnetic field has different orientations (angle $\varphi$ with the $y$-axis).

[Figure 9 about here]

Fig. 9. Contour plots of the velocity field (left) and the induced magnetic field (right) for Hartmann number $M=500$, electrode length $l=0.3$, wall inductance $k=1$ and magnetic field imposition angle (a) $\varphi=\pi / 3$ and (b) $\varphi=\pi / 6$. 
Fig. 9 shows contour plots of the velocity field and the induced magnetic field at steady state for Hartmann number $M=500$, by considering different orientations for the applied magnetic field. At steady state, both the velocity and the induced magnetic field reach a plateau, which tends to align with the direction of the applied magnetic field. The plateau for the case of velocity field is a stagnation region, with the recirculation zone increased in width as the angle of the applied field increases.

\subsection{Illustration of the proposed scheme for MHD flow in a duct with circular cross-section having insulating walls}

As a final example, we examine the flow in a long duct of circular cross-section with radius $R=1$. We consider variable wall conductivity, along with an applied magnetic field directed at angle $\varphi=\pi / 3$. Flow is driven downwards by electrodes placed in the top and bottom walls, with their start and bottom being at angle $\theta(\pi / 3 \leq \theta \leq 4 \pi / 3)$ and $(-\pi / 3 \leq \theta \leq-4 \pi / 3)$, where $\theta$ is the angle with respect of $x$-axis, while the remaining walls are kept at constant inductance, yet opposite in sign. The external magnetic field applied results in an induced current within the fluid, which can be forced to flow in the external circuits.

We use an irregular nodal distribution for low and moderate Hartmann numbers up to $M=1,000$. For Hartmann number $M=1,000$, we use local refinement close to the boundaries resulting in a nodal distribution which has 145,076 nodes (with this nodal distribution no oscillations were present) and can capture effectively the boundary layers. We solve the dynamic flow problem until reaching the steady-state solution. We use $k=1$ and the applied magnetic field angle $\varphi=\pi / 3$. Fig. 10 plots the velocity and induced magnetic field values for $M=1,000$.

[Figure 10 about here]

Fig. 10. Contour plots of the velocity (left) and the induced magnetic field (right) for Hartmann numbers $M=1,000$ at $\varphi=\pi / 3$.

\section{Conclusions}

In the present study, we present a robust, stable and accurate meshless point collocation method (MPCM) for the numerical solution of the coupled, unsteady MHD duct problem of an 
incompressible, viscous and electrically conducting fluid in a channel with partially conducting and partially non-conducting walls under a uniform transverse magnetic field. We solve the flow equations by using an explicit solver based on Euler time discretization method. Such a problem has many applications in industry and engineering and is interesting to get numerical solutions for high values of Hartmann number.

We solve the flow equations numerically by using the Discretization Corrected Particle Strength Exchange (DC PSE) approximation in the context of the meshless point collocation (MPC) method. The proposed method has several key advantages over traditionally used FEM, FDM or BEM methods: it requires neither domain nor surface mesh discretization, thus avoiding various topological, connectivity and dimensional difficulties associated with meshing; it does not require numerical integration; it is relatively easy to implement; the formulation is similar for $2 \mathrm{D}$ and $3 \mathrm{D}$ and both steady state and transient problems; and it is cost-effective due to the elimination of complex mesh creation steps.

We obtained numerical results using both uniform and irregular nodal distributions. We applied local refinement to capture the boundary layers formed at high Hartmann numbers. We developed an explicit solver, a simplified scheme for applying Neumann boundary conditions, and a method of estimating the maximum stable time step. We present numerical results for high Hartmann numbers (up to $M=5,000$ ) and compare them with analytical solutions and other well-established numerical methods. The numerical oscillations occurring due to the presence of boundary layers were eliminated by using a local refinement strategy.

\section{Acknowledgements:}

This research was supported in part by the Australian Government through the Australian Research Council's Discovery Projects funding scheme (project DP160100714). The views expressed herein are those of the authors and are not necessarily those of the Australian Government or Australian Research Council. We acknowledge the Raine Medical Research Foundation for funding of G. R. Joldes through a Raine Priming Grant.

\section{References}

[1] Hartmann J. (1937) Theory of the laminar flow of an electrically conductive liquid in a homogeneous magnetic field. Math Fys. Med;15 (6). 
[2] J Shercliff, Steady motion of conducting fluids in pipes under transverse magnetic fields," Mathematical Proceedings of the Cambridge Philosophical Society 49, 136-144 (1953).

[3] JCR Hunt, Magnetohydrodynamic flow in rectangular ducts," Journal of Fluid Mechanics 21, 577-590 (1965).

[4] JCR Hunt and K Stewartson, Magnetohydrodynamic flow in rectangular ducts. II," Journal of Fluid Mechanics 23, 563-581 (1965).

[5] J Hunt and W Williams, ISome electrically driven flows in magnetohydrodynamics. Part 1. Theory," Journal of Fluid Mechanics 31, 705-722 (1968).

[6] Gold R.R (1965), Magnetohydrodynamic pipe flow, Part 1, J. Fluid Mech. 21 577-590.

[7] J Baylis and J Hunt, MHD flow in an annular channel; theory and experiment," Journal of Fluid Mechanics 48, 423-428 (1971).

[8] P Tabeling and J Chabrerie, Magnetohydrodynamic secondary flows at high Hartmann numbers," Journal of Fluid Mechanics 103, 225-239 (1981).

[9] Singh B.; Lal J. MHD axial flow in a triangular pipe under transverse magnetic field, Indian J. Pure Appl. Math. 9 101-115 (1978).

[10] B. Singh, J. Lal, MHD axial flow in a triangular pipe under transverse magnetic field parallel to a side of the triangle, Indian J. Tech. 17 184-189 (1979).

[11] B. Singh, J. Lal, FEM in MHD channel flow problems, Internat. J. Numer. Methods Engrg. 18 1104-1111 (1982).

[12] B. Singh, J. Lal, FEM for unsteady MHD flow through pipes with arbitrary wall conductivity, Internat. J. Numer. Methods Fluids 4 291-302 (1984).

[13] Z. Demendy, T. Nagy, A new algorithm for solution of equations of mhd channel flows at moderate Hartmann numbers, Acta Mech. 123 135-149 (1997).

[14] M. Tezer-Sezgin, S. Koksal, FEM for solving MHD flow in a rectangular duct, Internat. J. Numer. Methods Engrg. 28 445-459 (1989).

[15] Shakeri, F.; Dehghan, M. A finite volume spectral element method for solving magnetohydrodynamic (MHD) equations, Applied Numerical Mathematics, Volume 61(1), (2011) Pages 1-23.

[16] M. Tezer-Sezgin, BEM solution of MHD flow in a rectangular duct, Internat. J. Numer. Methods Fluids 18 937-952 (1994).

[17] B. Singh, P. K. Agarwal, Numerical solution of a singular integral equation appearing in MHD. ZAMP 35:760-769 (1984).

[18] M. Tezer-Sezgin, S. H. Aydın, Dual reciprocity BEM for MHD flow using radial basis functions. Int J Comput Fluid Dyn 16:49-63 (2002). 
[19] Carabineanu A, Dinu A, Oprea I The application of the boundary element method to the magnetohydrodynamic duct flow. ZAMP 46:971-981 (1995).

[20] Hosseinzadeh, H.; Dehghan, M.; Mirzaei, D. The boundary elements method for magnetohydrodynamic (MHD) channel flows at high Hartmann numbers, Applied Mathematical Modeling, Volume 37(4), (2013) Pages 2337-2351.

[21] Liu, G.R., 2002. Mesh Free Methods, Moving beyond the Finite Elements Method, CRC Press.

[22] Gingold, R.A.; Monaghan, J.J. Smoothed particle hydrodynamics: theory and applications to non-spherical stars. Mon Not R Astron Soc, vol. 181, pp. 375-389 (1977).

[23] Nayroles, B; Touzot, G.; Villon, P, Generalizing the finite element method: diffuse approximation and diffuse elements. Comput Mech, vol. 10, pp. 307-318 (1992).

[24] Lu YY; Belytschko T.; Gu L. A new implementation of the element free Galerkin method. Computer Methods in Applied Mechanics and Engineering; 113:397-414 (1994).

[25] Liu WK.;Jun S; Zhang YF. Reproducing kernel particle methods. International Journal for Numerical Methods in Fluids; 20:1081-1106 (1995).

[26] Liu WK; Jun S; Li S, Adee J;Belytschko T. Reproducing kernel particle methods for structural dynamics. International Journal for Numerical Methods in Engineering; 38:16551679 (1995).

[27] Melenk JM, Babuska I. The partition of unity finite element method: basic theory and applications. Computer Methods in Applied Mechanics and Engineering 1996; 139:289-314 (1996).

[28] Duarte CA, Oden JT. An $h$ - $p$ adaptive method using clouds. Computer Methods in Applied Mechanics and Engineering 1996; 139:237-262 (1996).

[29] Liu W.K.; Li S.; Belytschko T. Moving least square reproducing kernel methods (I) methodology and convergence. Computer Methods in Applied Mechanics and Engineering 143:422-433 (1996).

[30] Zhu T.; Zhang J.; Atluri S.N. A meshless local boundary integral equation (LBIE) method for solving nonlinear problems. Computational Mechanics 22:174-186 (1998).

[31] Atluri S.N.; Kim H.G.; Cho J.Y. A critical assessment of the truly Meshless local PetrovGalerkin (MLPG) methods. Computational Mechanics 24:348-372 (1999).

[32] Atluri S.N.; Shen S.P. (2002) The Meshless Local Petrov-Galerkin (MLPG) Method, Tech Science Press, Encino USA.

[33] Onate E.; Idelsohn S.; Zienkiewicz O.; Taylor R.L., A finite point method in computational mechanics application to convective transport and fluid flow, Int. J. Numer. Methods Eng. 39 3839-3866 (1995). 
[34] Aluru NR. A point collocation method based on reproducing kernel approximations. International Journal for Numerical Methods in Engineering 2000; 47:1083-1121(2000).

[35] Fairweather F.; Karageorghis A. The method of fundamental solutions for elliptic boundary value problems, Adv. Comput. Math. 9, (1-2) 69-95 (1998).

[36] Fox L.; Henrici P.; Moler C. B. Approximations and bounds for eigenvalues of elliptic operators, SIAM J. Numer. Anal. 4 89-102 (1967).

[37] Brackbill, J.U., FLIP MHD: A Particle-in-Cell method for Magnetohydrodynamics, J. Comput. Phys., 96, pp. 163-192 (1991).

[38] Jiang, F., Oliveira, M.S.A., and Sousa, A.C.M., SPH simulation of transition to turbulence for planar shear flow subjected to a streamwise magnetic field, J. Comput. Phys., 217, pp. 485$501(2006)$.

[39] Verardi, S.L.L., Machado, J.M., and Shiyou, Y., The application of interpolating MLS approximations to the analysis of MHD flows, Fin Elem Anal Design, 39, pp. 1173-1187 (2003).

[40] Bourantas, G.C., Skouras, E.D., Loukopoulos, V.C., and Nikiforidis, G.C., An accurate, stable and efficient domain-type meshless method for the solution of MHD flow problems, J. Comput. Phys., 228, pp. 8135-8160 (2009).

[41] V.C. Loukopoulos, G.C. Bourantas, E.D. Skouras, G.C. Nikiforidis, , Localized meshless point collocation method for time-dependent magnetohydrodynamics flow through pipes under a variety of wall conductivity conditions Comput Mech 47:137-159 (2011).

[42] Dehghan, M., and Mirzaei, D., Meshless local boundary integral equation (LBIE) method for the unsteady magnetohydrodynamic (MHD) flow in rectangular and circular pipes, Comput. Phys. Commun., 180 1458-1466 (2009).

[43] Dehghan, M., and Mirzaei, D., Meshless local Petrov_Galerkin (MLPG) method for the unsteady magnetohydrodynamic (MHD) flow through pipe with arbitrary wall conductivity_, Appl. Numer. Math., 59 1043-1058 (2009).

[44] Dehghan, M., and Salehi, R., A meshfree weak-strong (MWS) form method for the unsteady magnetohydrodynamic (MHD) flow in pipe with arbitrary wall conductivity, Comput Mech., 52 1445-1462 (2013).

[45] Dehghan, M.; Mohammadi, V. The method of variably scaled radial kernels for solving two-dimensional magnetohydrodynamic (MHD) equations using two discretizations: The Crank-Nicolson scheme and the method of lines (MOL). Computers and Mathematics with Applications, Volume 70(10), (2015) Pages 2292-2315.

[46] Dehghan, M.; Haghjoo-Saniji, M. The local radial point interpolation meshless method for solving Maxwell equations. Engineering with Computers, Volume 33 (2017) Pages 897918. 
[47] Bourantas G.C.; Skouras E.D.; Nikiforidis G.C. Adaptive support domain implementation on the Moving Least Squares approximation for Mfree methods applied on elliptic and parabolic PDE problems using strong-form description, CMES: Comput. Model. Engng. 43 125 (2009).

[48] Kim D.W.; Liu W.K. Maximum principle and convergence analysis for the meshfree point collocation method", Siam J Numer Anal. 44 515-539 (2006).

[49] Dragos L.(1975) Magneto-fluid Dynamics. Academic Press: England

[50] Schrader, B., Reboux, S., Sbalzarini, I. F., Discretization correction of general integral PSE operators for particle methods. Journal of Computational Physics 229, 4159-4182 (2010).

[51] G. C. Bourantas, B. L. Cheesman, R. Ramaswamy, I. F. Sbalzarini. Using DC PSE operator discretization in Eulerian meshless collocation methods improves their robustness in complex geometries. Computers \& Fluids 136 285-300 (2016).

[52] Degond, P., Mas-Gallic, S. The weighted particle method for convection-diffusion equations. Part 2: The anisotropic case. Mathematics of Computation 53 (188), 509-525 (1989).

[53] Eldredge, J. D., Leonard, A., Colonius, T. A general deterministic treatment of derivatives in particle methods. Journal of Computational Physics 180 (2), 686-709 (2002).

[54] Schrader, B., 2011. Discretization-corrected PSE operators for adaptive multiresolution particle methods. Ph.D. thesis, Diss., Eidgenossische Technische Hochschule ETH Zurich, Nr. 19566, 2011.

[55] Isaacson, E. and Keller, H. B. 1966. Analysis of Numerical Methods, New York, Wiley, Library of Congress Catalog Card Number: 66-17630.

[56] Y. T. Gu, G. R. Liu. Meshless techniques for convection dominated problems. Comput. Mech. (2006) 38, 171-182.

[57] Sherliff JA, (1953) The motion of conducting fluids in pipes under transverse fields. Proceedings of the Cambridge Philosophical Society, vol. 49, 1953, 136-144.

[58] A.I. Nesliturk, M. Tezer-Sezgin, The finite element method for MHD flow at high Hartmann numbers, Comput. Meth. Appl. Mech. Eng. 194 (2005) 1201-1224.

Table 1. Critical time step for increasingly denser nodal distributions and for Hartmann number $\mathrm{M}=100,500$ and 1000 .

\begin{tabular}{cccccc}
\hline $\mathbf{M}$ & $\mathbf{8 1} \times \mathbf{8 1}$ & $\mathbf{1 6 1} \times \mathbf{1 6 1}$ & $\mathbf{3 2 1} \times \mathbf{3 2 1}$ & $\mathbf{6 4 1} \times \mathbf{6 4 1}$ & $\mathbf{1 2 8 1} \times \mathbf{1 2 8 1}$ \\
\hline 100 & & $3.17 \mathrm{e}-05$ & $9.48 \mathrm{e}-06$ & $2.57 \mathrm{e}-06$ & $6.84 \mathrm{e}-07$ \\
500 & $1.38 \mathrm{e}-05$ & $5.42 \mathrm{e}-06$ & $1.85 \mathrm{e}-06$ & $5.67 \mathrm{e}-07$ \\
1000 & $8.08 \mathrm{e}-06$ & $3.49 \mathrm{e}-06$ & $1.34 \mathrm{e}-06$ & $4.66 \mathrm{e}-07$ \\
\hline
\end{tabular}


Table 2a. The flow field and the magnetic field for $M=100$.

\begin{tabular}{ccccccc}
\hline & $\boldsymbol{V}_{\text {DCPSE }}$ & $\boldsymbol{V}_{\boldsymbol{F E M}}$ & $\boldsymbol{V}_{\text {analytical }}$ & $\boldsymbol{B}_{\text {DCPSE }}$ & $\boldsymbol{B}_{\text {FEM }}$ & $\boldsymbol{B}_{\text {analytical }}$ \\
\hline & & & & & & \\
$(0.00,0.00)$ & 0.0099999 & 0.0100000 & 0.0100000 & 0.0000000 & 0.0000000 & 0.0000000 \\
$(0.25,0.00)$ & 0.0099999 & 0.0100000 & 0.0100000 & -0.0024999 & -0.0025000 & -0.0025000 \\
$(0.50,0.00)$ & 0.0099999 & 0.0100000 & 0.0100000 & -0.0049999 & -0.0050000 & -0.0050000 \\
$(0.75,0.00)$ & 0.0099999 & 0.0100000 & 0.0100000 & -0.0074999 & -0.0075000 & -0.0075000 \\
$(0.00,0.25)$ & 0.0099999 & 0.0100000 & 0.0100000 & 0.0000000 & 0.0000000 & 0.0000000 \\
\hline
\end{tabular}




\begin{tabular}{ccccccc}
\hline$(0.25,0.25)$ & 0.0099999 & 0.0100000 & 0.0100000 & -0.0024999 & -0.0025000 & -0.0025000 \\
$(0.50,0.25)$ & 0.0099999 & 0.0099999 & 0.0100000 & -0.0049999 & -0.0050000 & -0.0050000 \\
$(0.75,0.25)$ & 0.0099999 & 0.0099999 & 0.0099999 & -0.0074999 & -0.0074999 & -0.0074999 \\
$(0.00,0.50)$ & 0.0099992 & 0.0099993 & 0.0099992 & 0.0000000 & 0.0000000 & 0.0000000 \\
$(0.25,0.50)$ & 0.0099982 & 0.0099983 & 0.0099981 & -0.0024982 & -0.0024984 & -0.0024982 \\
$(0.50,0.50)$ & 0.0099945 & 0.0099947 & 0.0099944 & -0.0049945 & -0.0049947 & -0.0049944 \\
$(0.75,0.50)$ & 0.0099869 & 0.0099873 & 0.0099868 & -0.0074869 & -0.0074873 & -0.0074868 \\
$(0.00,0.75)$ & 0.0097627 & 0.0097662 & 0.0097614 & 0.0000000 & 0.0000000 & 0.0000000 \\
$(0.25,0.75)$ & 0.0097175 & 0.0097209 & 0.0097163 & -0.0023033 & -0.0023043 & -0.0023030 \\
$(0.50,0.75)$ & 0.0095869 & 0.0095898 & 0.0095858 & -0.0046030 & -0.0046050 & -0.0046024 \\
$(0.75,0.75)$ & 0.0093872 & 0.0093899 & 0.0093863 & -0.0068877 & -0.0068903 & -0.0068869 \\
\hline
\end{tabular}

Table 2b. The flow field and the magnetic field for $M=500$.

\begin{tabular}{ccccccc}
\hline & $V_{D C P S E}$ & $\boldsymbol{V}_{\text {FEM }}$ & $\boldsymbol{V}_{\text {analytical }}$ & $\boldsymbol{B}_{\boldsymbol{D C P S E}}$ & $\boldsymbol{B}_{\boldsymbol{F E M}}$ & $\boldsymbol{B}_{\text {analytical }}$ \\
\hline & & & & & & \\
$(0.00,0.00)$ & 0.0020000 & 0.0020000 & 0.0020000 & 0.0000000 & 0.0000000 & 0.0000000 \\
$(0.25,0.00)$ & 0.0020000 & 0.0020000 & 0.0020000 & -0.0005000 & -0.0005000 & -0.0005000 \\
$(0.50,0.00)$ & 0.0020000 & 0.0020000 & 0.0020000 & -0.0009999 & -0.0010000 & -0.0010000 \\
$(0.75,0.00)$ & 0.0020000 & 0.0020000 & 0.0020000 & -0.0014999 & -0.0015000 & -0.0015000 \\
$(0.00,0.25)$ & 0.0020000 & 0.0020000 & 0.0020000 & 0.0000000 & 0.0000000 & 0.0000000 \\
$(0.25,0.25)$ & 0.0020000 & 0.0020000 & 0.0020000 & -0.0005000 & -0.0005000 & -0.0005000 \\
$(0.50,0.25)$ & 0.0020000 & 0.0020000 & 0.0020000 & -0.0009999 & -0.0010000 & -0.0010000 \\
$(0.75,0.25)$ & 0.0020000 & 0.0020000 & 0.0020000 & -0.0014999 & -0.0015000 & -0.0015000 \\
$(0.00,0.50)$ & 0.0020000 & 0.0020000 & 0.0020000 & 0.0000000 & -0.0000000 & 0.0000000 \\
$(0.25,0.50)$ & 0.0020000 & 0.0020000 & 0.0020000 & -0.0005000 & -0.0005000 & -0.0005000 \\
$(0.50,0.50)$ & 0.0019999 & 0.0020000 & 0.0020000 & -0.0009999 & -0.0010000 & -0.0010000 \\
$(0.75,0.50)$ & 0.0019999 & 0.0020000 & 0.0020000 & -0.0014999 & -0.0015000 & -0.0015000 \\
$(0.00,0.75)$ & 0.0019999 & 0.0020000 & 0.0020000 & 0.0000000 & 0.0000000 & 0.0000000 \\
$(0.25,0.75)$ & 0.0019999 & 0.0019999 & 0.0019999 & -0.0004999 & -0.0005000 & -0.0004999 \\
$(0.50,0.75)$ & 0.0019997 & 0.0019998 & 0.0019997 & -0.0009997 & -0.0009980 & -0.0009997 \\
$(0.75,0.75)$ & 0.0019992 & 0.0019994 & 0.0019992 & -0.0014992 & -0.0014994 & -0.0014992 \\
& & & & & & \\
\hline
\end{tabular}

Table 2c. Convergence for the flow field for $M=500$ and successively denser grids.

\begin{tabular}{lllllll}
\hline & $\mathbf{8 1} \times \mathbf{8 1}$ & $\mathbf{1 6 1 \boldsymbol { x } 1 6 1}$ & $\mathbf{3 2 1 \boldsymbol { x } 3 \mathbf { 1 }}$ & $\mathbf{6 4 1 \boldsymbol { x } 6 4 1}$ & $\mathbf{1 2 8 1 \boldsymbol { x } 1 2 8 1}$ & $\boldsymbol{V}_{\text {analytical }}$ \\
\hline & & & & & & \\
$(0.00,0.00)$ & 0.0019999 & 0.0020000 & 0.0020000 & 0.0020000 & 0.0020000 & 0.0020000 \\
$(0.25,0.00)$ & 0.0019996 & 0.0020000 & 0.0020000 & 0.0020000 & 0.0020000 & 0.0020000 \\
$(0.50,0.00)$ & 0.0019925 & 0.0019999 & 0.0020000 & 0.0020000 & 0.0020000 & 0.0020000 \\
$(0.75,0.00)$ & 0.0018415 & 0.0019999 & 0.0020000 & 0.0020000 & 0.0020000 & 0.0020000 \\
$(0.00,0.25)$ & 0.0019999 & 0.0020000 & 0.0020000 & 0.0020000 & 0.0020000 & 0.0020000 \\
$(0.25,0.25)$ & 0.0019996 & 0.0020000 & 0.0020000 & 0.0020000 & 0.0020000 & 0.0020000 \\
$(0.50,0.25)$ & & 0.0019999 & 0.0020000 & 0.0020000 & 0.0020000 & 0.0020000 \\
\hline
\end{tabular}




\begin{tabular}{lllllll}
\hline$(0.75,0.25)$ & 0.0019924 & 0.0019999 & 0.0020000 & 0.0020000 & 0.0020000 & 0.0020000 \\
$(0.00,0.50)$ & 0.0018519 & 0.0020000 & 0.0020000 & 0.0020000 & 0.0020000 & 0.0020000 \\
$(0.25,0.50)$ & 0.0019999 & 0.0020000 & 0.0020000 & 0.0020000 & 0.0020000 & 0.0020000 \\
$(0.50,0.50)$ & 0.0019996 & 0.0019999 & 0.0019999 & 0.0019999 & 0.0019999 & 0.0020000 \\
$(0.75,0.50)$ & 0.0019929 & 0.0019999 & 0.0019999 & 0.0019999 & 0.0019999 & 0.0020000 \\
$(0.00,0.75)$ & 0.0018474 & 0.0019999 & 0.0019999 & 0.0019999 & 0.0019999 & 0.0020000 \\
$(0.25,0.75)$ & 0.0019999 & 0.0019999 & 0.0019999 & 0.0019999 & 0.0019999 & 0.0019999 \\
$(0.50,0.75)$ & 0.0019996 & 0.0019997 & 0.0019997 & 0.0019997 & 0.0019997 & 0.0019997 \\
$(0.75,0.75)$ & 0.0019931 & 0.0019992 & 0.0019992 & 0.0019992 & 0.0019992 & 0.0019992 \\
& 0.0018567 & & & & & \\
\hline
\end{tabular}

Table 3a. The flow field for $M=1,000$ at different time instances.

\begin{tabular}{cccccc}
\hline & $\boldsymbol{t}=\mathbf{0 . 0 2}$ & $\boldsymbol{t}=\mathbf{0 . 0 4}$ & $\boldsymbol{t}=\mathbf{0 . 1}$ & $\boldsymbol{t}=\mathbf{0 . 2}$ & $\boldsymbol{t}=\mathbf{0 . 4}$ \\
\hline & & & & & \\
$(0.00,0.00)$ & 1.0233522 & 1.0192380 & 0.9955148 & 1.0002997 & 1.0000002 \\
$(0.25,0.00)$ & 0.9934001 & 0.9798223 & 0.9996844 & 1.0002481 & 0.9999997 \\
$(0.50,0.00)$ & 0.9937160 & 1.0177319 & 1.0006521 & 1.0003009 & 1.0000000 \\
$(0.75,0.00)$ & 0.9771426 & 1.0001986 & 0.9964664 & 1.0005023 & 1.0000025 \\
$(0.00,0.25)$ & 1.0233515 & 1.0192365 & 0.9955157 & 1.0002995 & 1.0000001 \\
$(0.25,0.25)$ & 0.9934002 & 0.9798238 & 0.9996844 & 1.0002479 & 0.9999997 \\
\hline
\end{tabular}




\begin{tabular}{llllll}
\hline$(0.50,0.25)$ & 0.9937164 & 1.0177302 & 1.0006519 & 1.0003008 & 1.0000000 \\
$(0.75,0.25)$ & 0.9771433 & 1.0001985 & 0.9964670 & 1.0005021 & 1.0000025 \\
$(0.00,0.50)$ & 1.0233510 & 1.0192356 & 0.9955161 & 1.0002994 & 1.0000001 \\
$(0.25,0.50)$ & 0.9934006 & 0.9798248 & 0.9996846 & 1.0002479 & 0.9999997 \\
$(0.50,0.50)$ & 0.9937159 & 1.0177292 & 1.0006522 & 1.0003008 & 1.0000000 \\
$(0.75,0.50)$ & 0.9771441 & 1.0001982 & 0.9964674 & 1.0005019 & 1.0000025 \\
$(0.00,0.75)$ & 1.0233506 & 1.0192350 & 0.9955079 & 1.0003101 & 1.0000005 \\
$(0.25,0.75)$ & 0.9934005 & 0.9798255 & 0.9996841 & 1.0002567 & 0.9999993 \\
$(0.50,0.75)$ & 0.9937171 & 1.0177277 & 1.0006547 & 1.0003087 & 0.9999997 \\
$(0.75,0.75)$ & 0.9771439 & 1.0002000 & 0.9964638 & 1.0005143 & 1.0000039 \\
\hline
\end{tabular}

Table 3b. The magnetic field for $M=2,000$ at different time instances.

\begin{tabular}{cccccc}
\hline & $\boldsymbol{t}=\mathbf{0 . 0 2}$ & $\boldsymbol{t}=\mathbf{0 . 0 4}$ & $\boldsymbol{t}=\mathbf{0 . 1}$ & $\boldsymbol{t}=\mathbf{0 . 2}$ & $\boldsymbol{t}=\mathbf{0 . 4}$ \\
\hline & & & & & \\
$(0.00,0.00)$ & 0.0000000 & -0.0000000 & 0.0000000 & 0.0000000 & 0.0000000 \\
$(0.25,0.00)$ & -0.0320597 & -0.0020130 & -0.0030101 & 0.0000000 & 0.0000003 \\
$(0.50,0.00)$ & 0.0384021 & -0.0039995 & -0.0029465 & -0.0003524 & 0.0000000 \\
$(0.75,0.00)$ & -0.0051009 & 0.0098014 & 0.0033888 & -0.0005073 & -0.0000025 \\
$(0.00,0.25)$ & 0.0000000 & -0.0000000 & 0.0000000 & 0.0000000 & 0.0000000 \\
$(0.25,0.25)$ & -0.0320587 & -0.0020128 & -0.0030094 & -0.0001011 & 0.0000003 \\
$(0.50,0.25)$ & 0.0384009 & -0.0039991 & -0.0029459 & -0.0003523 & 0.0000000 \\
$(0.75,0.25)$ & -0.0051008 & 0.0098008 & 0.0033881 & -0.0005071 & -0.0000025 \\
$(0.00,0.50)$ & 0.0000000 & 0.0000000 & 0.0000000 & 0.0000000 & 0.0000000 \\
$(0.25,0.50)$ & -0.0320578 & 0.0020130 & -0.0030090 & -0.0001010 & 0.0000003 \\
$(0.50,0.50)$ & 0.0383998 & -0.0039995 & -0.0029455 & -0.0003522 & 0.0000000 \\
$(0.75,0.50)$ & -0.0051004 & 0.0098001 & 0.0033878 & -0.0005070 & -0.0000025 \\
$(0.00,0.75)$ & 0.0000000 & 0.0000000 & 0.0000000 & 0.0000000 & 0.0000000 \\
$(0.25,0.75)$ & -0.0320571 & 0.0020128 & -0.0030122 & -0.0001029 & 0.0000007 \\
$(0.50,0.75)$ & 0.0383979 & -0.0039984 & -0.0029495 & -0.0003625 & 0.0000002 \\
$(0.75,0.75)$ & -0.0050999 & 0.0097979 & 0.0033912 & -0.0005192 & -0.0000039 \\
\hline
\end{tabular}




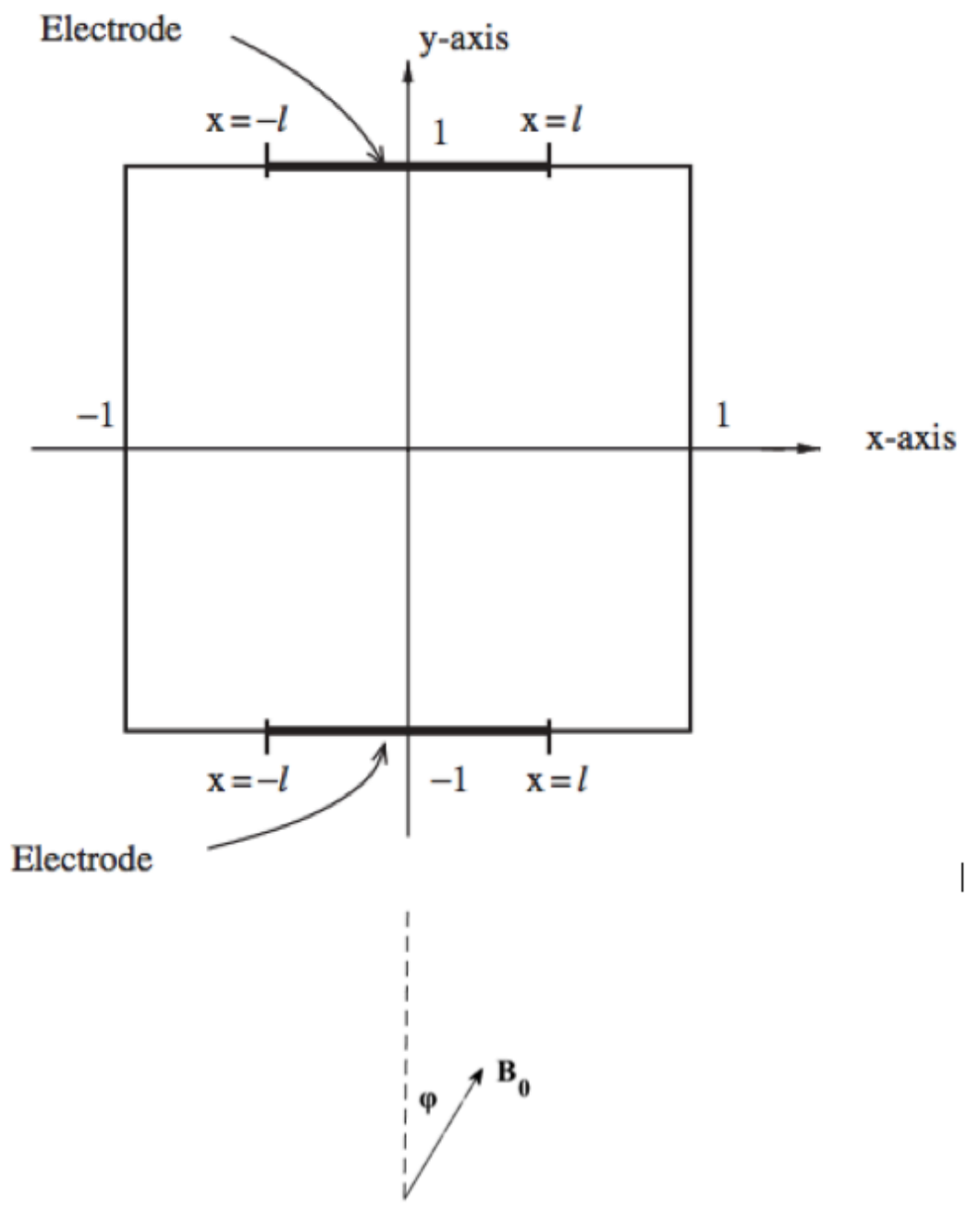

Fig.1 
Fig. 2 


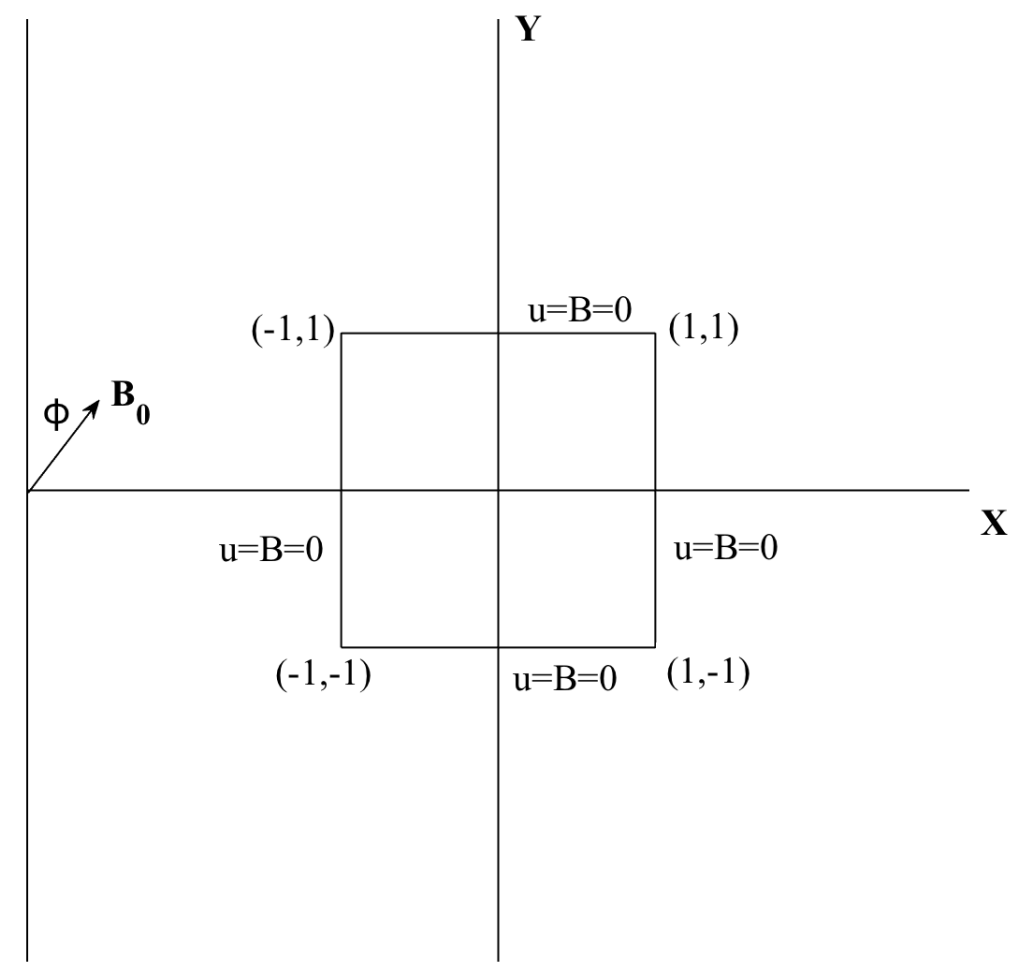

Fig. 3 


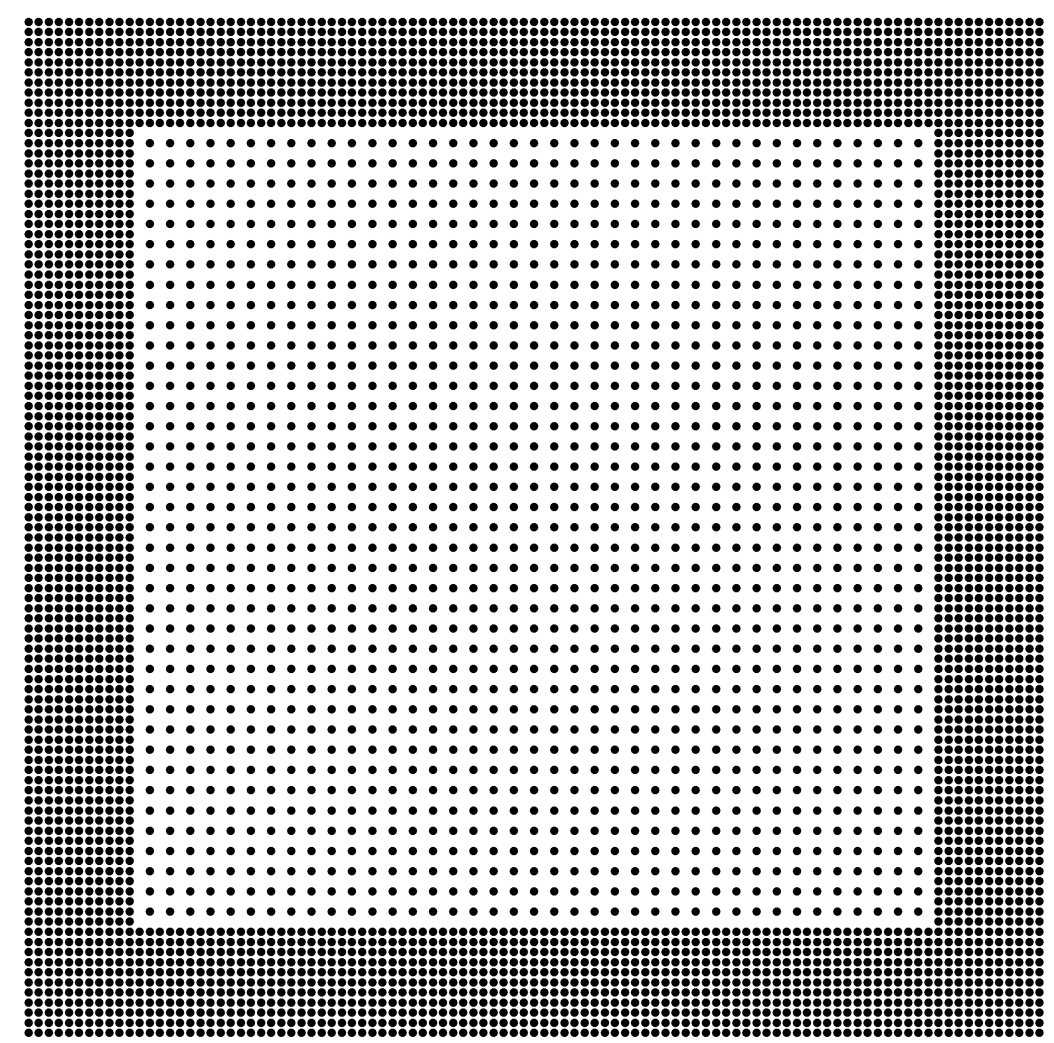

(a)

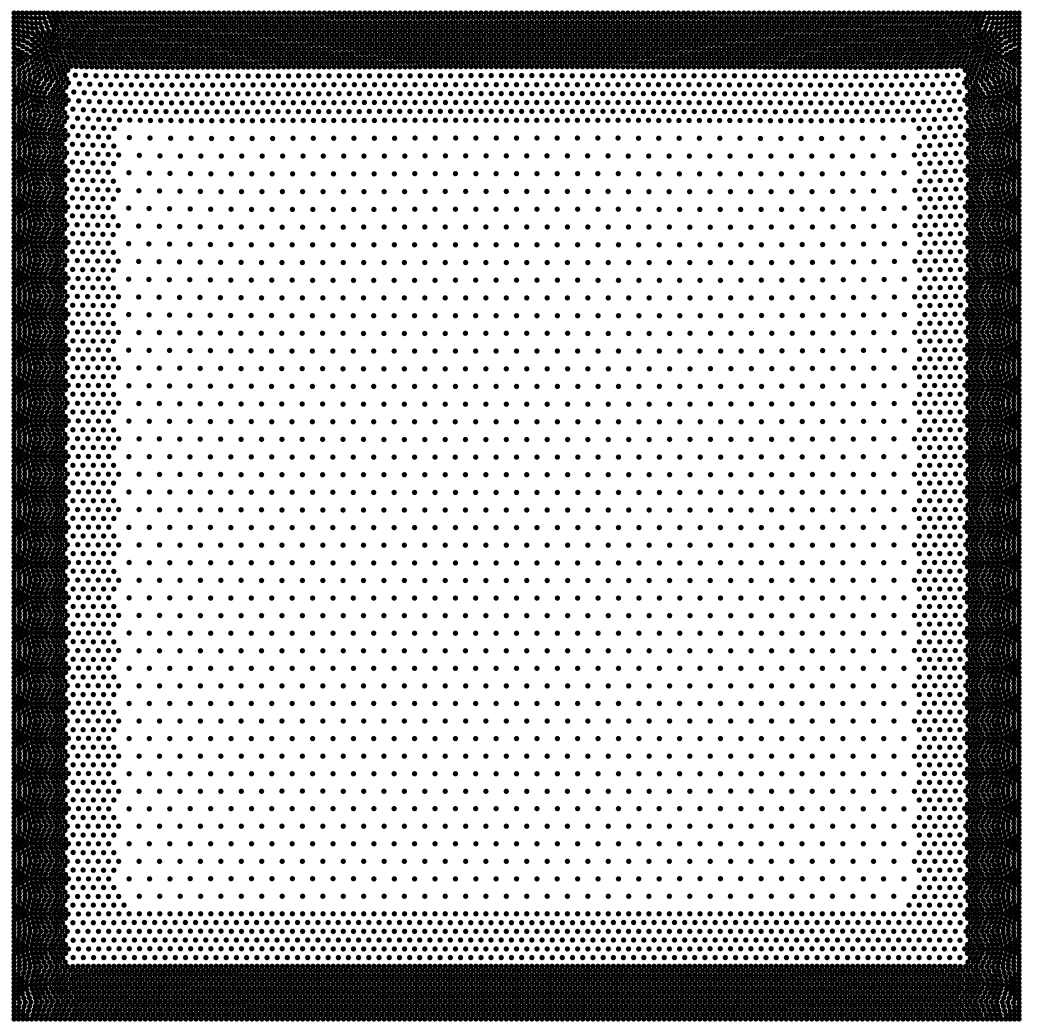

(b)

Fig. 4 

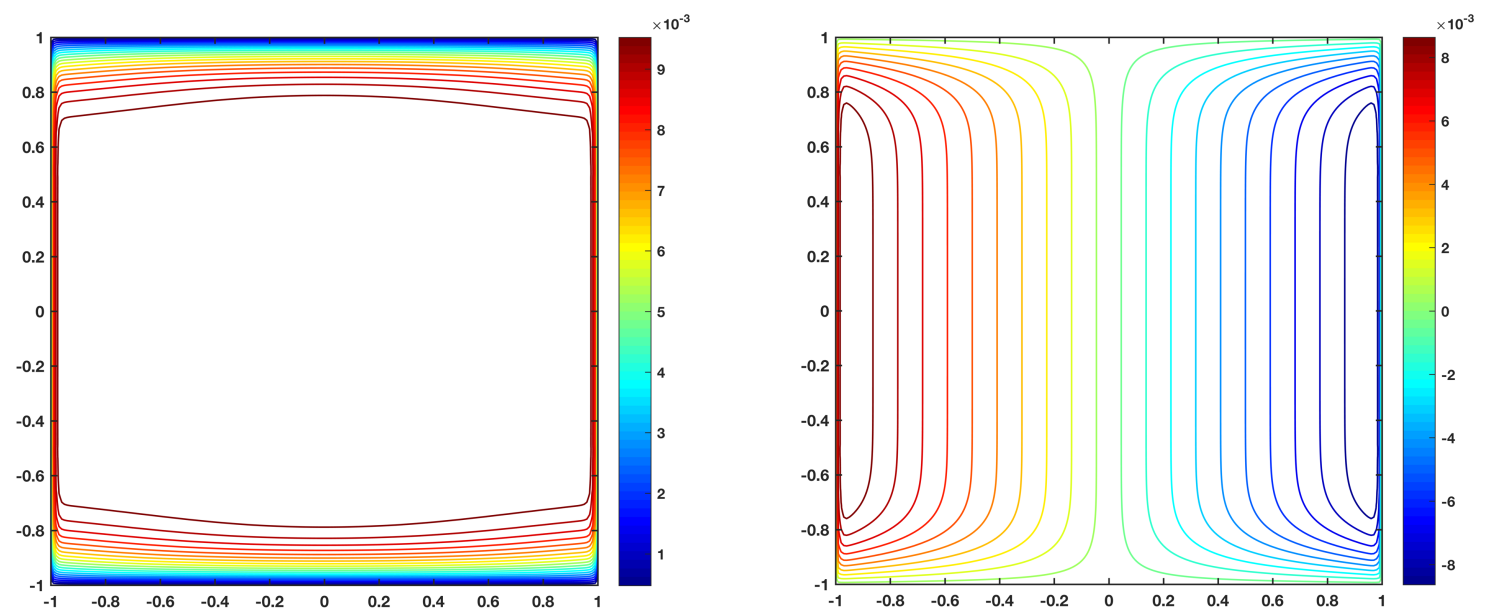

(a)
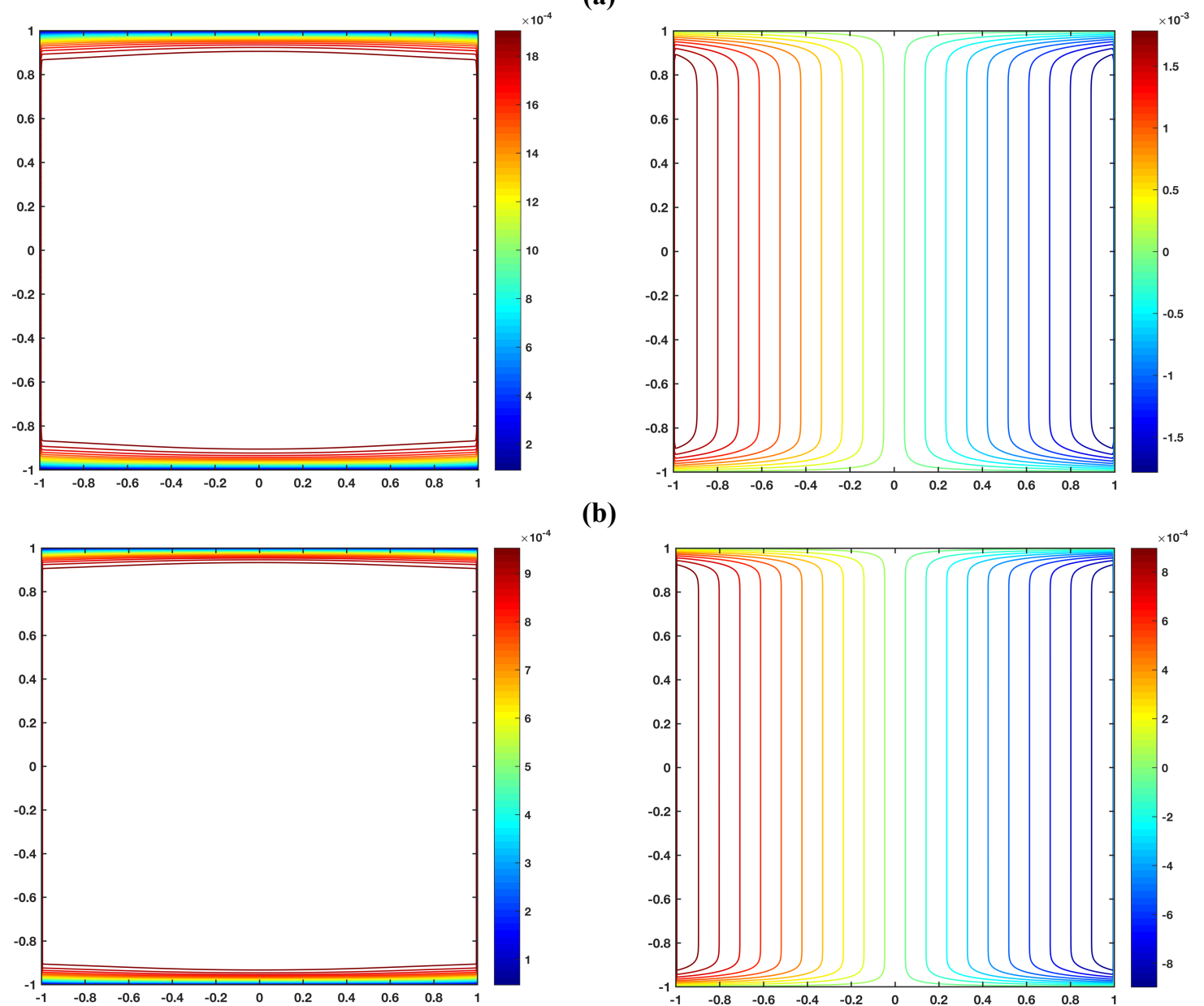

(b)

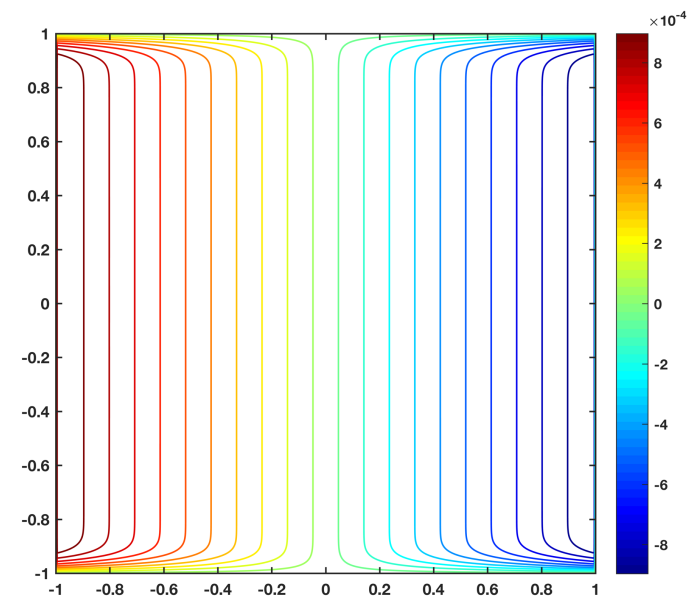

(c)

Fig. 5 

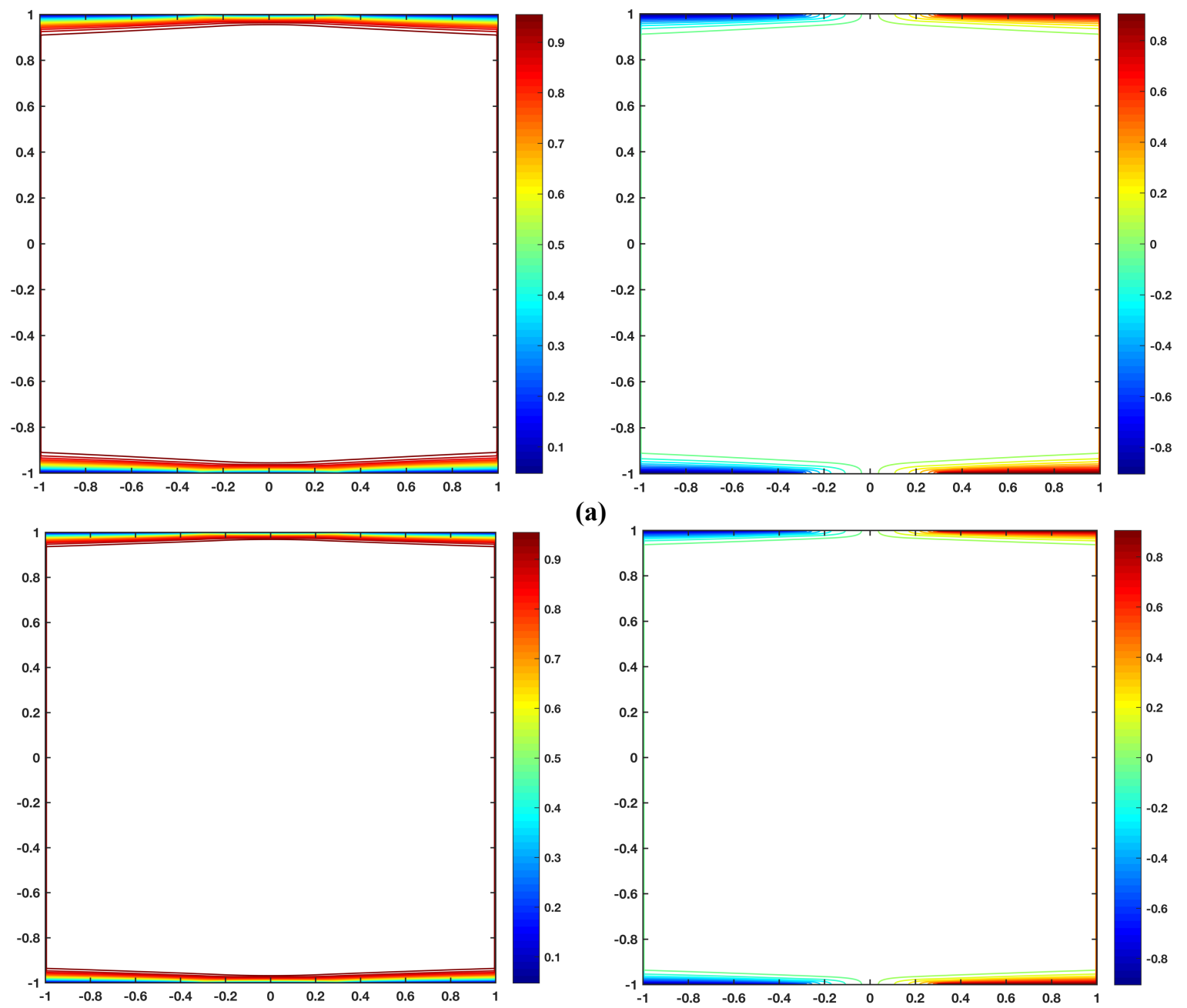

(a)

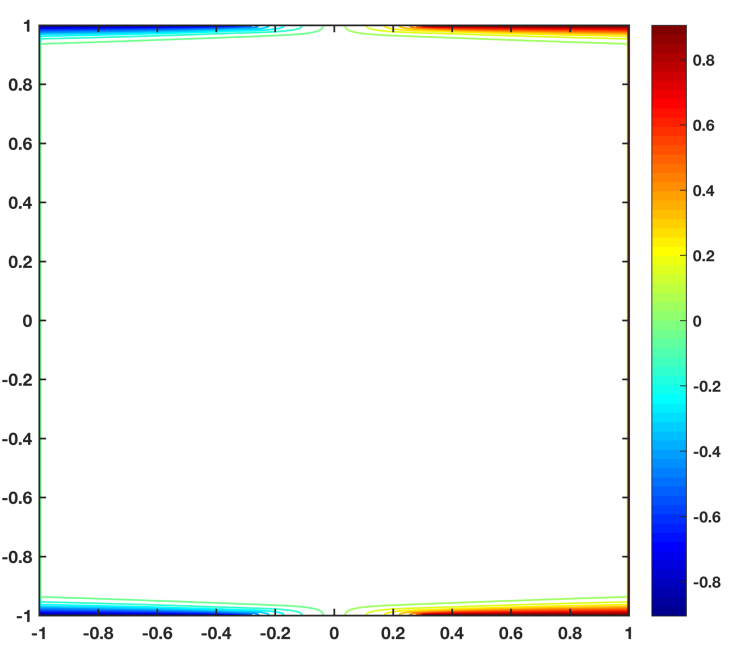

(b)

Fig. 6 

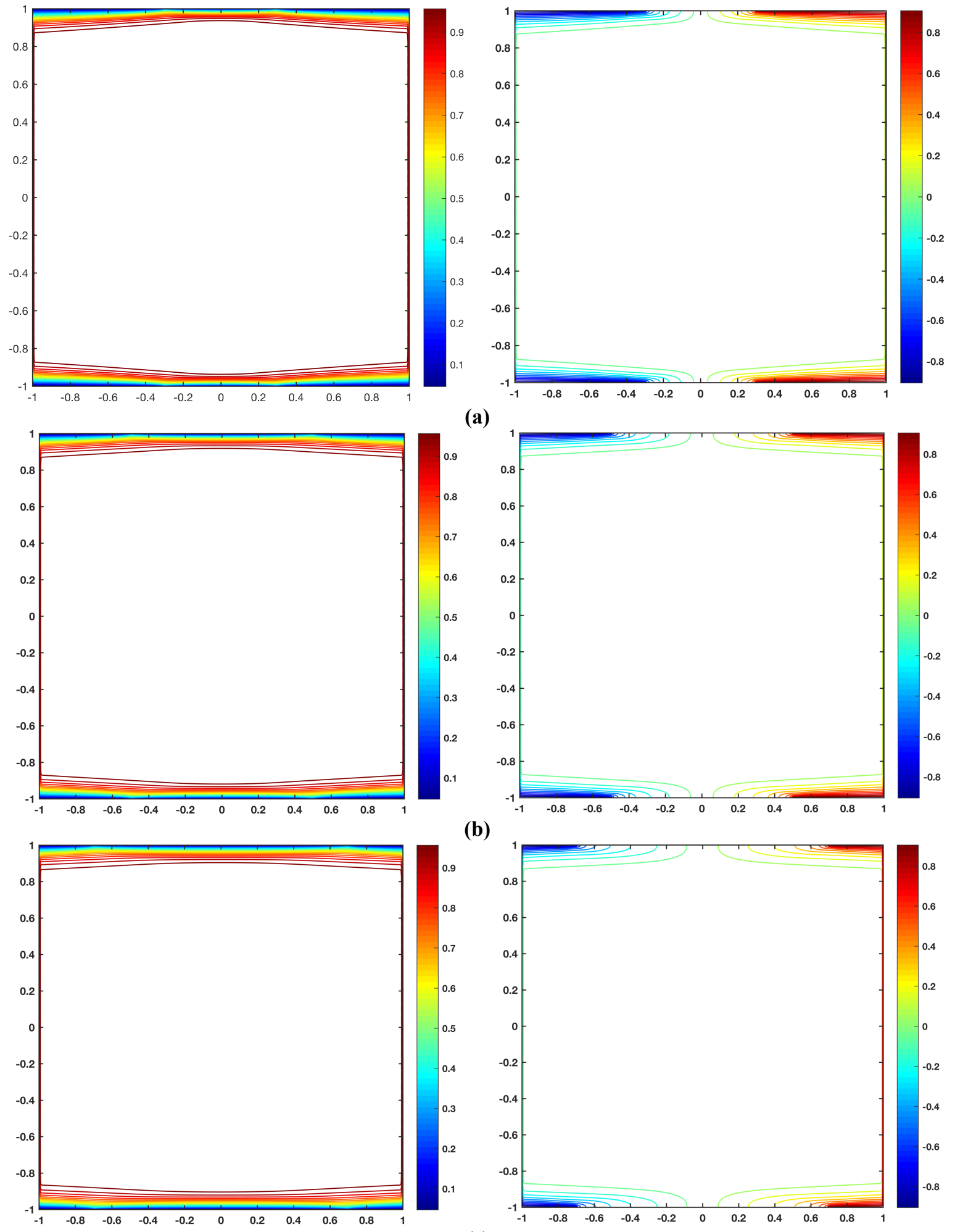

(b)

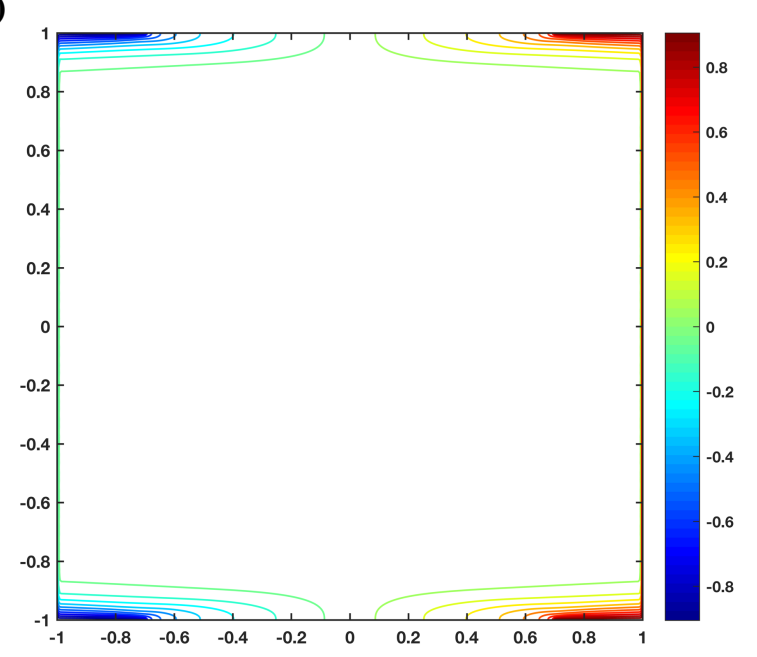

(c)

Fig. 7 

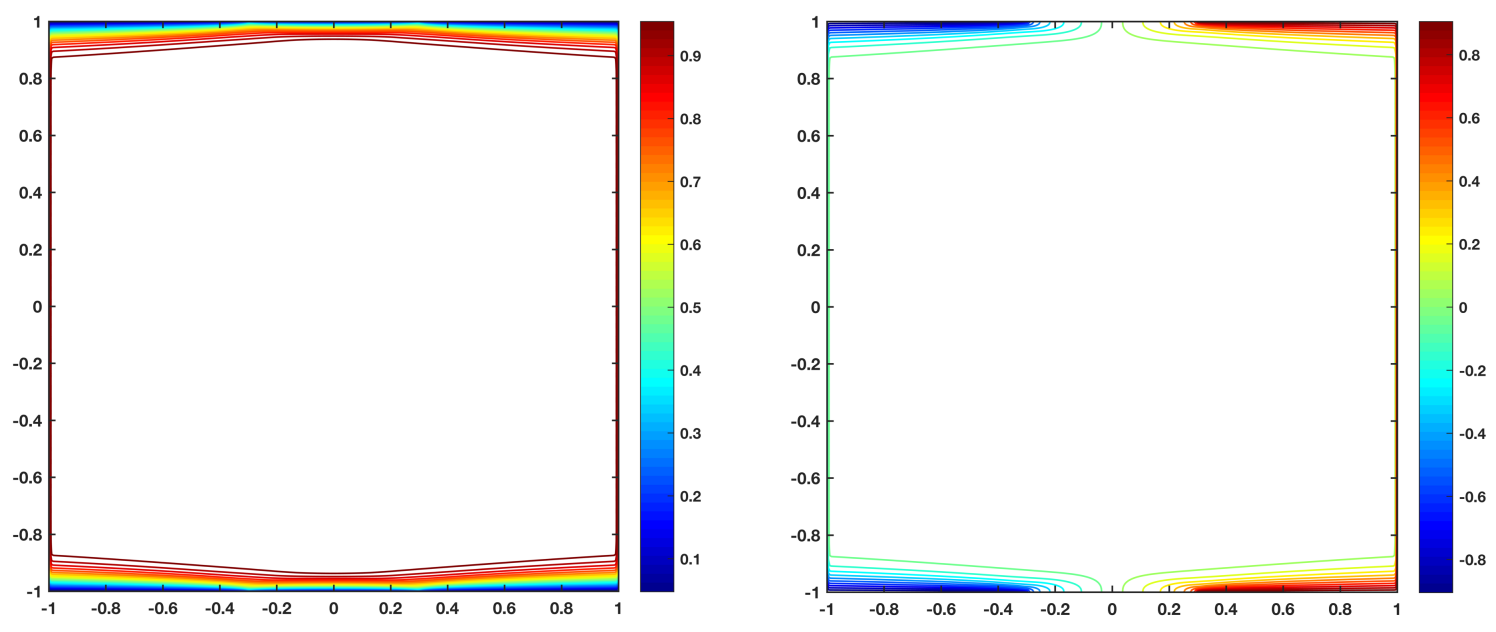

(a)
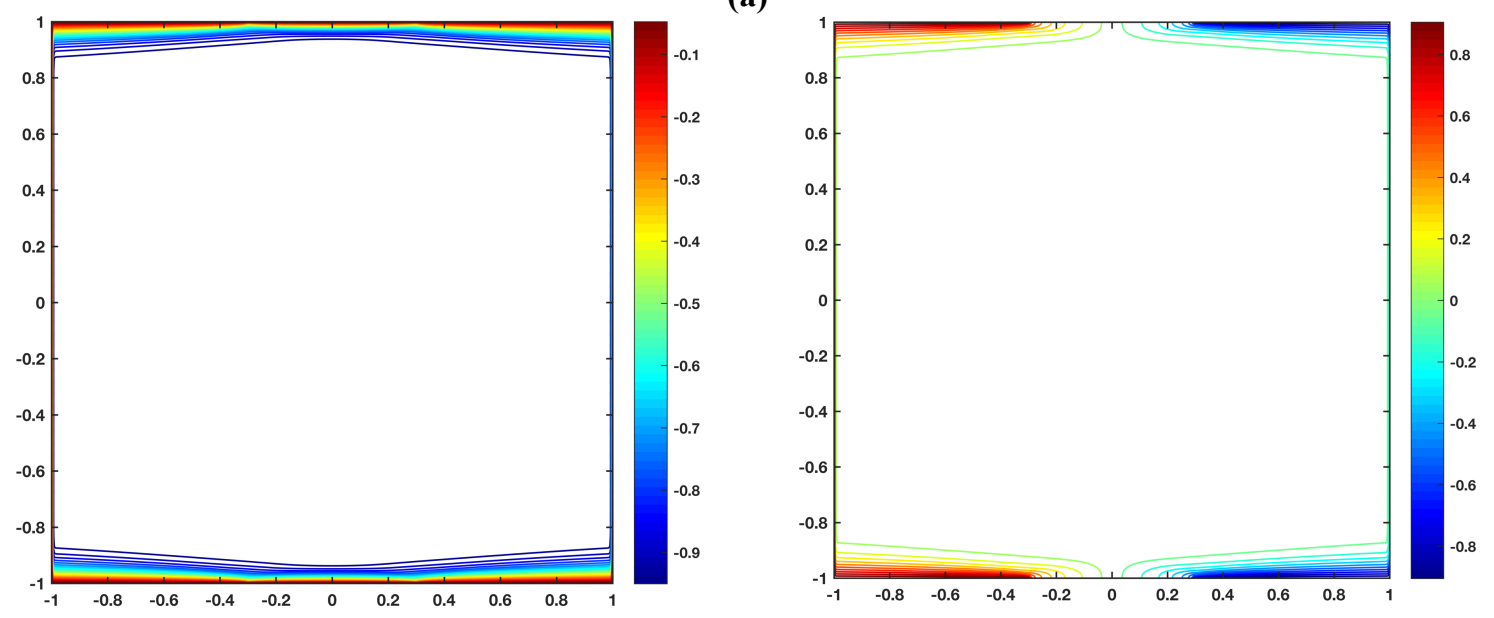

(b)
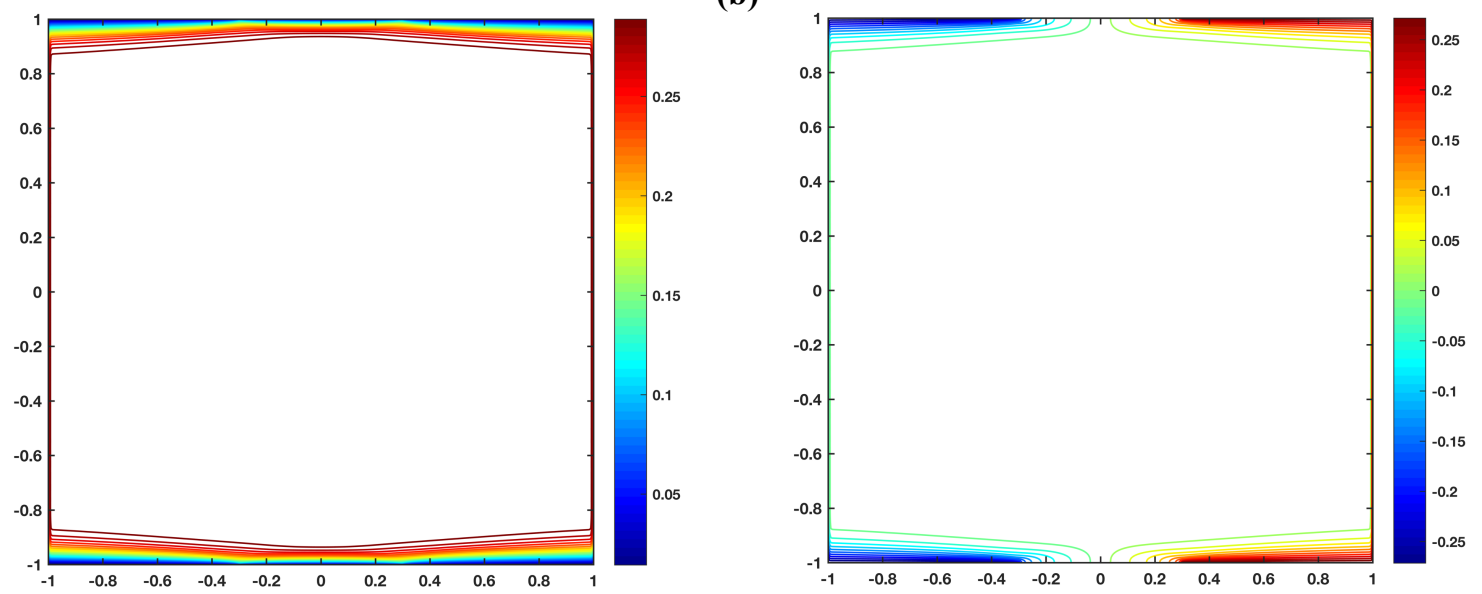

(c)

Fig. 8 

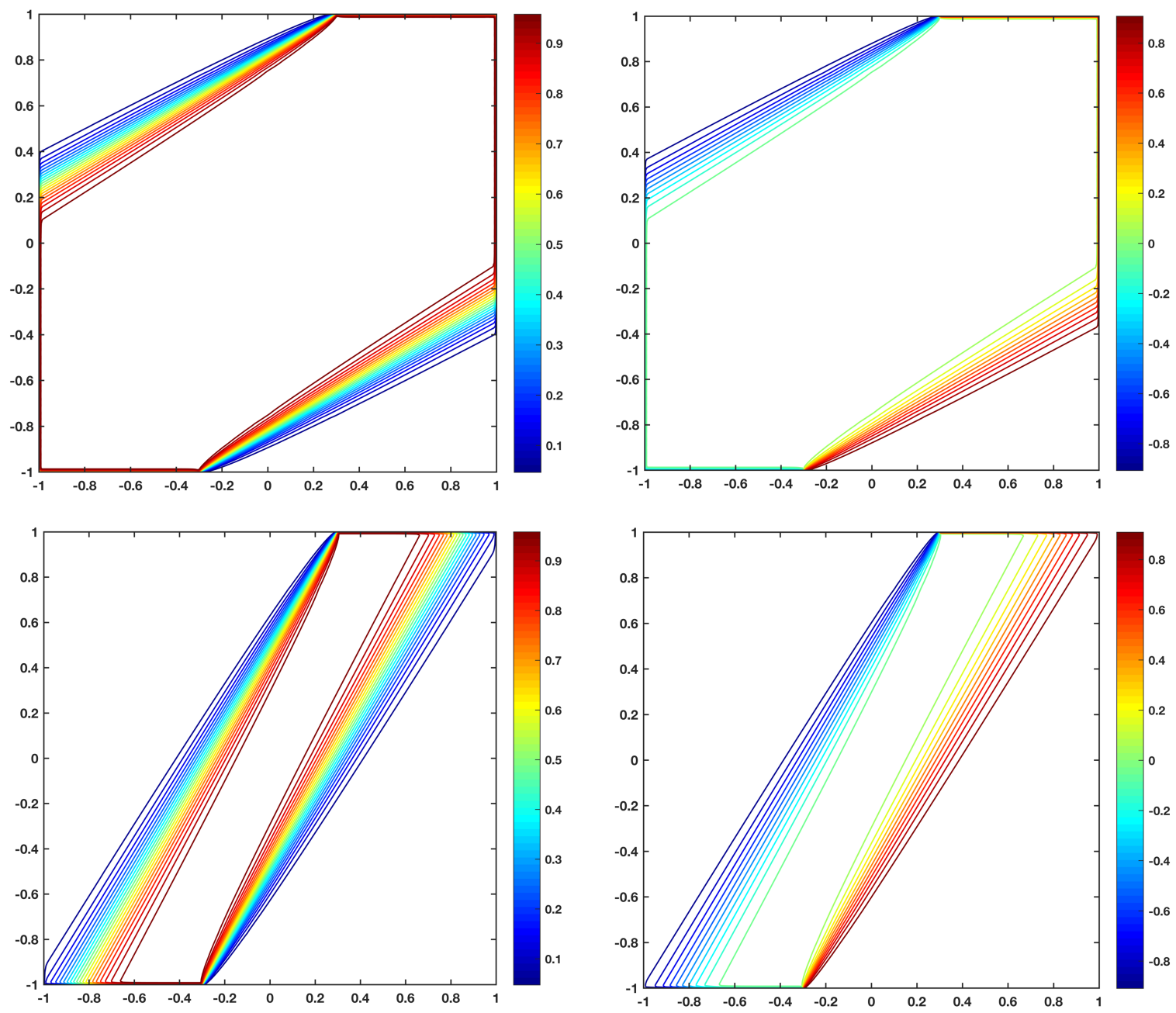

(b)

Fig. 9 


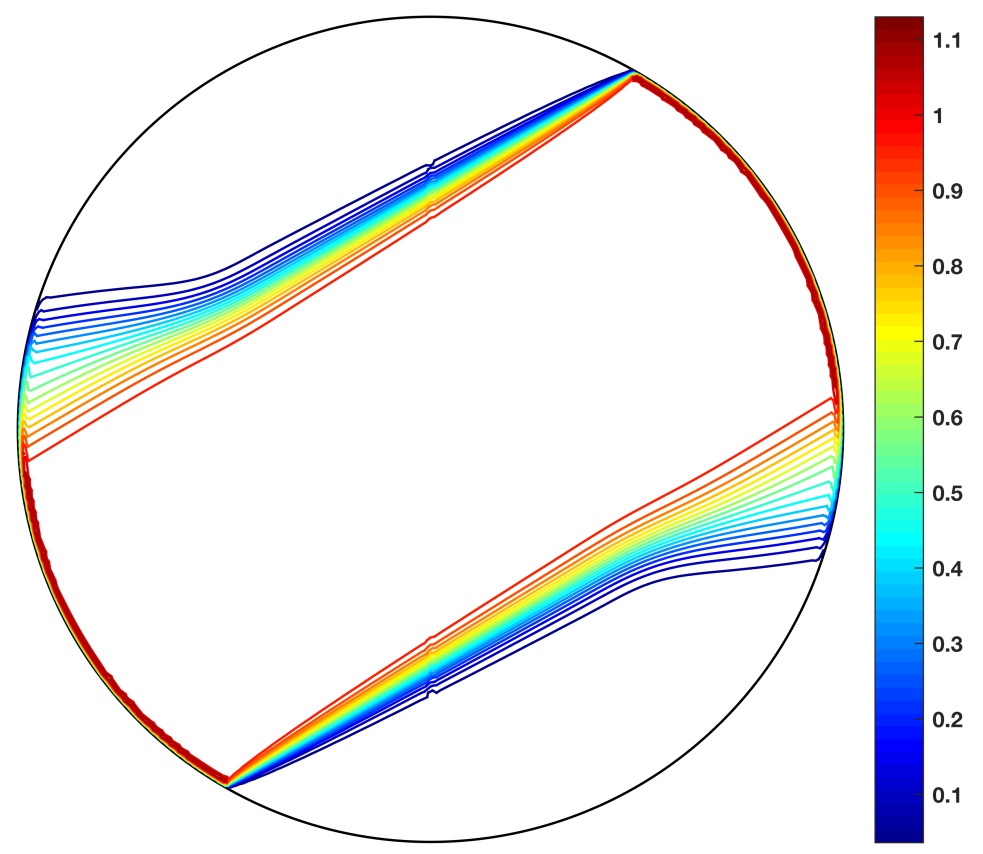

(a)

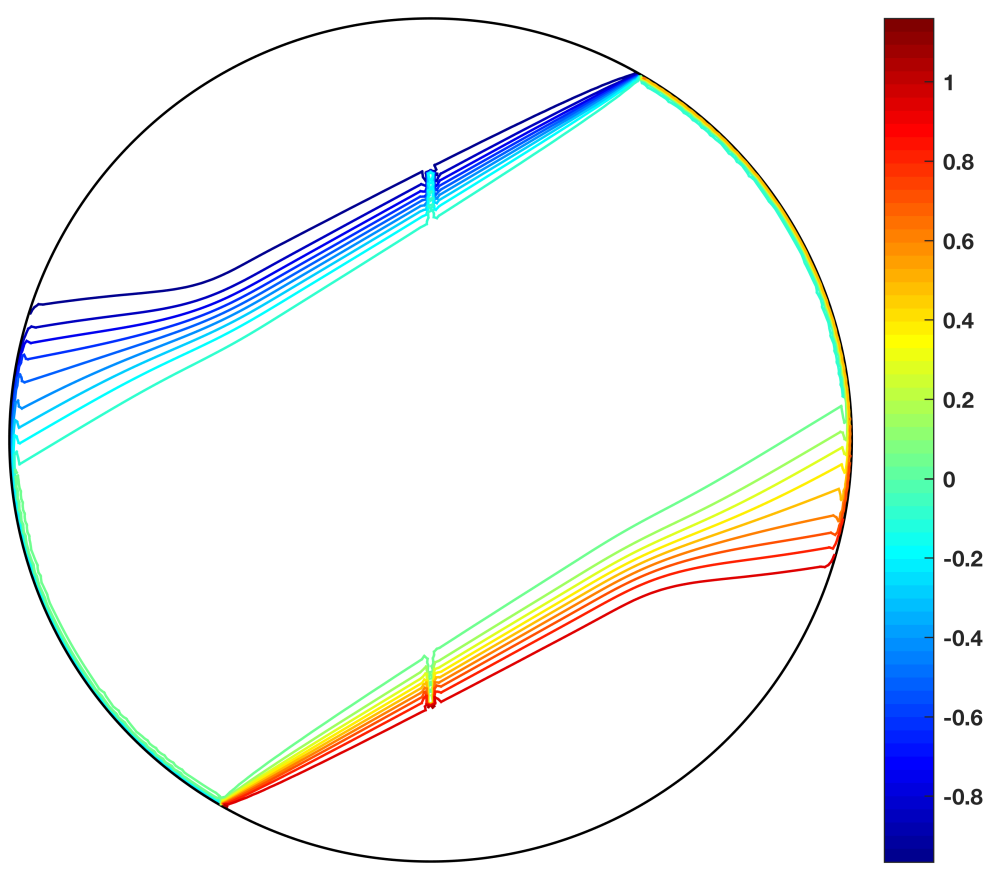

(b)

Fig. 10 